\title{
Rheology modification in mixed shape colloidal dispersions. Part I: pure components
}

\author{
Annemieke J. W. ten Brinke, ${ }^{a}$ Louise Bailey, ${ }^{b}$ Henk N. W. Lekkerkerker ${ }^{a}$ and Geoffrey C. Maitland ${ }^{* c}$ \\ Received 29th March 2007, Accepted 1st June 2007 \\ First published as an Advance Article on the web 12th July 2007 \\ DOI: 10.1039/b704742h
}

The flow behaviour and rheology of colloidal dispersions are of considerable interest in many applications, for example colloidal clay particles find applications in oilfield and constructiondrilling fluids. The rheological properties of such fluids can be enhanced significantly by adding colloidal particles of different size and shape. To gain insight into the mechanism of this phenomenon, we have studied model mineral-colloid systems whose shape changes systematically from a plate-like aluminasol (gibbsite), through a lath-like smectite clay (hectorite), to a rod-like aluminasol (boehmite).

The paper presents the results of a systematic and comprehensive multi-technique study (oscillatory, transient and steady shear) of the rheology of dispersions of these model systems. This gives a detailed account of the 'yield space' that characterises the complex transition of these soft materials from elastoviscous gels to viscoelastic liquids, and of the effect of particle size and shape on this behaviour. The observed phenomena are underpinned by two competing flowmediated microstructural rearrangements that have significantly different timescales. A physical model invoking flow-mediated building and disruption of fluid structure is described to rationalise the observed behaviour. The study also forms the baseline to a companion study (part II), which investigates the rheological behaviour of mixed anisometric colloid systems based on these pure components.

\section{Introduction}

This paper is concerned with the rheological properties of dispersions of highly non-spherical colloidal particles. Anisometric colloids, especially natural clays, are widely used in a range of applications, from personal care products to oilwell drilling. ${ }^{1-3}$ These exploit one or more of several functionalities typical of clay particles. They are capable of giving relatively high viscosity suspensions at low volume fractions (and low shear rates) due to the highly effective space-filling capability of rotating anisotropic particles such as platelets and needles, in addition to any specific particle interactions. This high low-stress viscosity is usually accompanied by a significant (effective, if not real) yield stress, $\tau_{\mathrm{y}}$, or static elastic (storage) modulus, $G_{0}$. This gelation to form a soft solid is a reflection of a highly interactive particle-network structure whose interaction energies are large compared with thermal energies. However, once the imposed mechanical stresses start to exceed the interparticle forces, the suspensions become highly shear-thinning, so that this high viscosity at low shear rates/stresses reduces significantly as the imposed stress or strain rate is increased. It is often highly time-dependent before attaining a steady-state value characteristic of the

\footnotetext{
${ }^{a}$ Van't Hoff Laboratory for Physical and Colloid Chemistry, Utrecht University, Padualaan 8, 3584 CH, Utrecht, The Netherlands

${ }^{b}$ Schlumberger Cambridge Research, High Cross, Madingley Road, Cambridge, UK CB3 OEL

${ }^{c}$ Department of Chemical Engineering, Imperial College London, South Kensington, London, UK SW7 $2 A Z$
}

balance of imposed stresses and Brownian forces. This occurs because of time-dependent disruption of the relatively weakly interacting particle-network structure, and alignment of the anisometric species involved. On cessation or reduction in flow, the gel structure rebuilds through Brownian motion such that the stress at any given shear rate, $\dot{\gamma}$, is a function of time; $\tau_{\mathrm{y}}$ and $G_{0}$ are therefore time-dependent quantities.

This thixotropy is a characteristic that is strongly exploited in many applications, ${ }^{4}$ where a material is required to behave as an immobile solid in some situations but needs to be able to flow easily in others. A key requirement in designing such materials is the ability to tune the rate and intensity of these thixotropic transformations. Some applications require a rapid change from liquid to solid-like behaviour e.g. toothpaste, where rapidly gelling laponite clays have been used. Others require long recovery times, for example to allow the soft solid to take up a new shape e.g. adhesives or particle coatings. The intensity and timescales of the structural changes underpinning colloidal dispersion thixotropy and their resulting rheological effects are highly dependent on the nature of the clay particles: their size and shape, the distribution of these, and the specific particle interactions due to electrostatic, van der Waals dispersion and depletion effects. The differences between clay particles can be quite subtle. For instance, kaolin and montmorillonite particles are both platelets possessing negative charges on both edges and faces above their respective isoelectric points. However, at the same volume fraction the latter forms much stronger gels and displays far greater 
thixotropy. This reflects differences in platelet size, aspect ratio (diameter-thickness) and hence flexibility, surface-charge density amongst other factors. The detailed nature of the colloid particle is therefore crucial in designing thixotropic fluids for specific applications.

Wierenga and Philipse ${ }^{5}$ have reviewed theory and experiments of the low-shear viscosity of dispersions of anisotropic colloids. For dilute dispersions of rod-like particles, the dimensions of the rods largely determine the viscosity; at higher particle concentrations specific material properties strongly influence the low-shear viscosity. The same authors have also discussed ${ }^{5}$ the possible effect of particle shape on gel properties.

The major variables available to tune dispersion rheological properties are particle concentration and factors which mediate the strength of the particle interactions: the nature of the particles themselves, ionic strength for electrostatic interactions, added polymers (adsorbing for steric-stabilising repulsive interactions or for bridging long-range attractions, and non-absorbing for attractive depletion interactions). Recently there has been increased interest in how addition of a second colloid component of different size, shape or charge affects the properties of colloidal systems, both in their phase behaviour and their rheological properties. ${ }^{6-9}$ Lekkerkerker and co-workers ${ }^{10-12}$ have demonstrated that the phase behaviour of mixed platelet-rod-sphere systems is extremely rich. The kinetics and arrest of crystallisation, phase separation and appearance of amorphous, glass-like sediments in such mixed systems have also been studied. Rhodes and Lewis, ${ }^{13}$ Louis et al. ${ }^{14}$ and others have shown that small colloidal particles can give rise to both attractive and repulsive depletion interactions between larger colloids, depending on their relative size and charge.

There are several examples ${ }^{2,15}$ of industrial fluids where mixtures of colloids of different size, shape or charge have been used to modify the rheology. Yet the rheological properties and the underpinning particle-scale dynamics of mixed sizeshape colloid systems have been relatively little studied. The aim of the current research is to better understand the behaviour of more complex industrial fluids by studying the thixotropic behaviour of suspensions of a series of wellcharacterised model colloid systems, and to demonstrate the range of behaviour observed when colloids of different shape and/or size are mixed. Whilst much has been published on the rheology of thixotropic suspensions, ${ }^{16,17}$ including clay dispersions, ${ }^{3,18-22}$ with the development of phenomenological models, which attempt to characterise the thixotropic behaviour using parameters to describe the extent and rate of 'structure' build-up and breakdown, ${ }^{23}$ relatively little is known about how to control the magnitude and timescales of the rheological changes through tuning of the particle interactions. The industrial formulation process still does this in a largely empirical manner. The development of this understanding, which can lead to systematic design rules, requires studies of rheology and dynamics to be performed on very wellcharacterised model colloids. The majority of such studies have been on spherical colloid systems, where the classical studies of Russel and Sperry ${ }^{24}$ and others ${ }^{25-28}$ have established a good understanding of microstructural-rheology relationships over the whole concentration range. This work is part of an activity aimed at bringing similar insight to the behaviour of non-dilute suspensions of well-characterised anisotropic colloidal particles and their mixtures.

For the current work, we have undertaken a comprehensive study of the effect of particle shape on the rheology of concentrated dispersions. There have been many studies of montmorillonite, a natural plate-like colloid widely used in industrial applications ${ }^{29}$ and for academic studies. ${ }^{21,30-34}$ Here we have chosen to focus on an alternative natural clay colloid, hectorite, which has similar application potential but has been studied in far less detail. The sample used has been well characterised at the particle level and can be considered to be a clean, model system. The particles are lath-like in shape - 'flat rods' with $d<w<L$. Its behaviour is compared with two other systems that represent shape modifications in two directions. Reducing the lath width, $w$, so that $d=w \ll L$, gives a rod-like particle, represented here by boehmite, whilst extending this dimension to be comparable with the length ( $d \ll w=L)$ gives platelets, in the form of another aluminasol, gibbsite. This paper presents a detailed comparison of the flow behaviour of these pure components. Whilst this provides the necessary base-line, against which the behaviour of mixed systems of the same components will be compared in a companion paper (part II), the pure component suspensions are of interest in their own right. A challenge for thixotropic, pseudo-yield stress fluids is to obtain a self-consistent and reproducible rheological description that covers their response to different flow types. Here it is shown that by using appropriate pre-treatment procedures, the behaviour of these suspensions in oscillatory, transient and steady shear flow can be reconciled by a simple viscoelastic model. The transition from elastic gel-like behaviour to viscous liquid-like flow turns out to be characterised by significantly more complex stressstrain-strain-rate relationships than have hitherto been associated with so-called 'yield stress' fluids. Parallels may be drawn with other self-assembling and shear-sensitive materials such as surfactant fluids, and the paper sets out to give both a characterisation methodology and a rheological framework for soft, highly shear-sensitive viscoelastic solids. An attempt is also made to relate the macroscopic rheology to the particlescale dynamics and interactions.

\section{Experimental}

\section{Materials: preparation and characterisation}

Hectorite, SHCa-1, was supplied by the Clay Minerals Society, Source Clays Repository. This hectorite has a cation-exchange capacity of $43.9 \mathrm{meq}$ per $100 \mathrm{~g}$ and a BET-surface area of $63.19 \mathrm{~m}^{2} \mathrm{~g}^{-1}$. The hectorite was further purified before use: the particles were dispersed and pre-hydrated in demineralised water for 48 hours, and subsequently centrifuged three times for 20 minutes at $400 \mathrm{G}$. After each centrifugation step the supernatant was collected, and the filtrate was redispersed in demineralised water and re-centrifuged. The particles of $>2 \mu \mathrm{m}$ in size were removed in this way by sedimentation. The suspension was dialysed against demineralised water for 14 days. TEM images of the hectorite particles, taken using a Technai 12 electron microscope, are shown in Fig. 1a. 


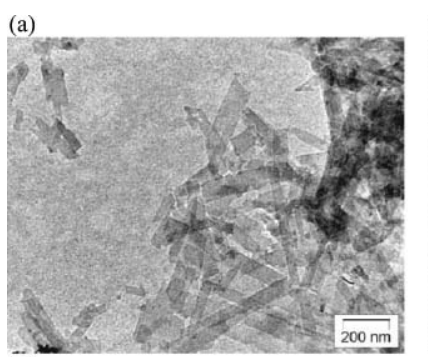

(b)
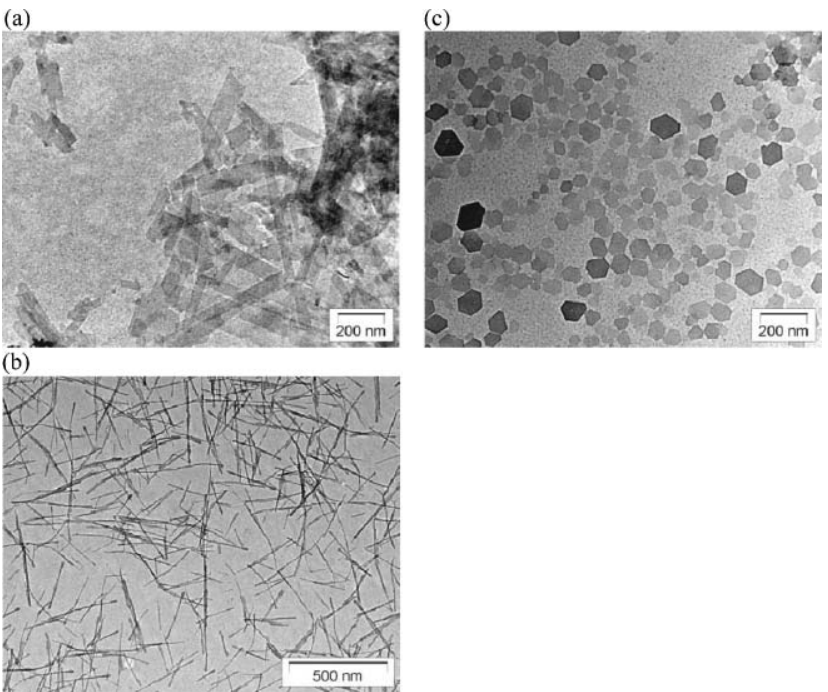

Fig. 1 Representative TEM images of (a) hectorite, (b) boehmite, and (c) gibbsite particles.

This clearly shows the lath-like nature of the hectorite particles. Their average length after sedimentation is $288 \mathrm{~nm}$ with a polydispersity of $47 \%$. The average width of the laths is $43 \mathrm{~nm}$, with a polydispersity of $24 \%$. These average dimensions were measured from 100 particles on several TEM micrographs.

Boehmite $^{35}$ and gibbsite ${ }^{36}$ were synthesised from aluminium tri-sec-butoxide and aluminium tri-isopropoxide using techniques developed in this laboratory. A mixture of $0.08 \mathrm{~mol}^{-1}$ aluminium tri-sec-butoxide, $0.08 \mathrm{~mol} \mathrm{l}^{-1}$ aluminium triisopropoxide and $0.09 \mathrm{~mol}^{-1}$ hydrochloric acid in demineralised water was aged for 7-10 days at room temperature under continuous stirring. The mixture was then heated in an autoclave, either for 72 hours at $85{ }^{\circ} \mathrm{C}$ to prepare gibbsite or for 22 hours at $150{ }^{\circ} \mathrm{C}$ to prepare boehmite. Suspensions of gibbsite and boehmite were dialysed for 14 days against demineralised water. TEM pictures of the boehmite and gibbsite particles are shown in Fig. 1b and 1c, respectively.

For the boehmite rods an average length of about $200 \mathrm{~nm}$ was determined, with a polydispersity of $44 \%$, and an average thickness of $9.4 \mathrm{~nm}$ (polydispersity 15\%). The average diameter of the gibbsite platelets was determined from the TEM images to be approximately $81 \mathrm{~nm}$ (polydispersity $26 \%$ ).

Atomic force microscopy, AFM, was also used to characterise the dimensions of the particles, in particular to give a better estimate of the thickness of the plates. The colloid particles were imaged in air with the Nanoscope IIIa (Multimode AFM, Digital Instruments). AFM measurements were carried out in tapping mode, where the cantilever oscillates close to resonance and the tip only slightly touches the surface. Standard TESP silicon tips (purchased from Digital Instruments) were used with a tip radius $<10 \mathrm{~nm}$. A drop of colloid suspension was released onto a freshly cleaved mica surface and dried in air. The results for boehmite and gibbsite are shown in Fig. 2a and 2b, respectively. Hectorite particles could not be characterised by AFM because they clustered on the mica substrate.

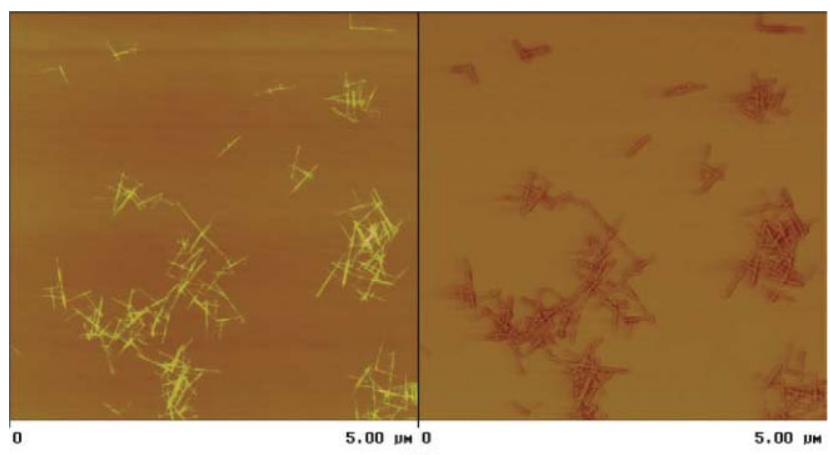

(a)

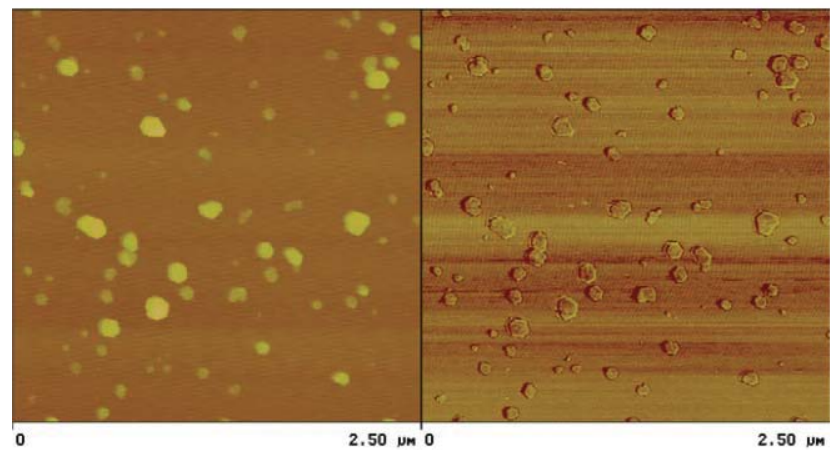

(b)

Fig. 2 (a) AFM images of boehmite particles: $5 \times 5 \mu \mathrm{m}$ plots of height (left) and phase (right) obtained in tapping mode. (b) AFM images of gibbsite particles: $2.5 \times 2.5 \mu \mathrm{m}$ plots of height (left) and phase (right) obtained in tapping mode.

The average length of the boehmite particles (determined from 50 particles on several AFM images) is $317 \mathrm{~nm}$, with a polydispersity of $28 \%$. Smaller particles are more difficult to see in an AFM image, because they are more difficult to separate from the background (the substrate surface) due to noise. This may be the reason for the longer average length of boehmite when compared to the TEM measurements. The thickness of the rods could not be determined from the AFM image because the particles lie over one another.

For gibbsite the average diameter determined from AFM images is $86 \mathrm{~nm}$, in good agreement with the TEM diameter. The average thickness is $5.6 \mathrm{~nm}$. Wierenga et al. ${ }^{36}$ estimated from TEM images a thickness of $14 \mathrm{~nm}$ for gibbsite particles with a diameter of $160 \mathrm{~nm}$, which is of the same order of magnitude to our particles, with a similar aspect ratio.

Table 1 gives a summary of the characteristics of the particles investigated. The length of the particles is designated $L_{1}$, the width $L_{2}$ and the thickness $d$. The thickness of the hectorite particles is assumed to be the same as for the gibbsite particles. The density was measured using an Anton Paar DMA 5000 density meter. The mobility of the pure gibbsite, boehmite and hectorite dispersions was measured using a Coulter DELSA (Doppler electrophoretic sonic amplitude) 440SX apparatus. The table contains a factor $\alpha$, which is defined as the ratio of the hydrodynamic (swept) volume $\left(4 \pi L_{1}{ }^{3} / 24\right)$ over the real volume of the particles $\left(\sim L_{1} L_{2} d\right)$. The critical concentration, $c^{*}$, which is the overlap concentration (at which the particles' hydrodynamic volumes start to overlap and so impose strong steric constraints on their neighbours) is 
Table 1 Particle characteristics of the model colloids studied in this work

\begin{tabular}{llll}
\hline Property & Hectorite & Gibbsite & Boehmite \\
\hline Shape & Lath & Plate & Rod \\
$L_{1} / \mathrm{nm}$ & 288 & 81 & 200 \\
$L_{2} / \mathrm{nm}$ & 43 & 81 & 10 \\
$d / \mathrm{nm}$ & 6 & $6^{a}$ & 10 \\
Density $/ \mathrm{g} \mathrm{cm}^{-3}$ & 2.39 & 1.96 & 2.06 \\
$\alpha^{b}$ & 160 & 6.8 & 187 \\
$c^{*} / \mathrm{g}$ per $100 \mathrm{~cm}^{3}$ & 1.5 & 28.8 & 1.1 \\
$\mathrm{pH}^{c}$ & 8.9 & 7.8 & 6.2 \\
Sign of face charge & - & + & + \\
Conductivity $/ \mu \mathrm{S} \mathrm{cm}$ & $38.1 @$ & $80.2 @$ & $46.0 @$ \\
& $1336 \mathrm{ppm}$ & $1154 \mathrm{ppm}$ & $1045 \mathrm{ppm}$ \\
Mobility $/ 10^{-8} \mathrm{~m}^{2} \mathrm{Vs}^{-1}$ & -1.2 & 2.8 & 3.9 \\
Zeta potential $/ \mathrm{mV}^{-1}$ & -9.8 & +24.0 & +44.2
\end{tabular}

${ }^{a}$ Determined from AFM, the other $d$ values are estimated from TEM. ${ }^{b}$ Ratio of hydrodynamic volume $V_{\mathrm{h}}$ to real particle volume ${ }^{c}$ Natural $\mathrm{pH}$ of suspensions as used in this study

calculated using eqn (1):

$$
\frac{c^{*}}{g \text { per } 100 \mathrm{~cm}^{3}}=\frac{100 L_{1} L_{2} 24 d \rho}{4 \pi L_{1}^{3}} \sim \frac{200 L_{2} d \rho}{L_{1}^{2}} \mathrm{wt} \%
$$

\section{Rheological measurements}

The rheology of the pure model systems, gibbsite, boehmite, and hectorite was characterised at two different concentrations, $\sim 1 \mathrm{wt} \%$ and $\sim 2.5 \mathrm{wt} \%$. All measurements were carried out at $20{ }^{\circ} \mathrm{C}$, without $\mathrm{pH}$ adjustment or salt addition. A Physica Anton Paar (MCR-300) controlled stress rheometer, with a cone-plate geometry, diameter of $5 \mathrm{~cm}$ and a cone angle of $1^{\circ}$, was used.

All the suspensions show thixotropic behaviour, reflecting the finite time taken to move from any one state of microstructure to another as a result of the competition between break-down of the interacting colloidal network due to flow stresses and its build-up due to flow-induced collisions and Brownian motion. Consequently, to ensure reproducibility, it was necessary to use a well-defined sample loading and pre-treatment protocol to ensure that the measurements were independent of sample history, and to wait for the

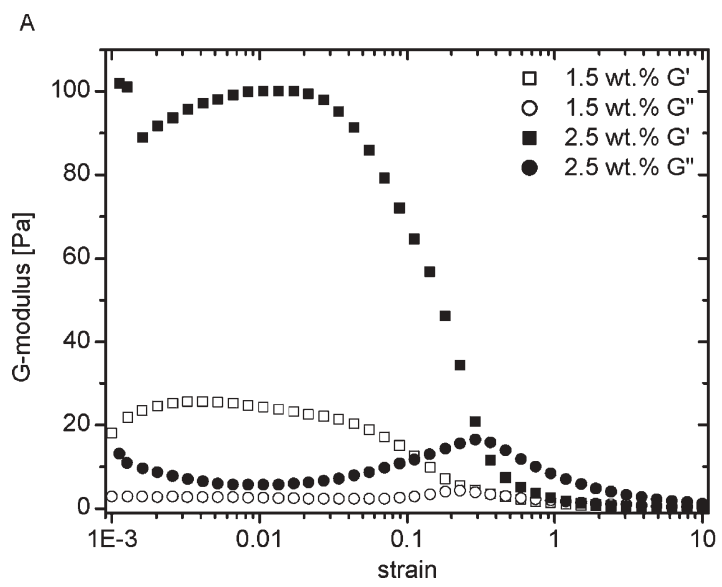

shear stress to come to a steady-state value at each imposed shear-rate. ${ }^{4}$

Two such pre-treatment protocols were adopted for steady shear measurements: (a) broken gel, $60 \mathrm{~s}$ pre-shear at a shear rate of $500 \mathrm{~s}^{-1}$; (b) recovered gel, $60 \mathrm{~s}$ pre-shear at a shear rate of $500 \mathrm{~s}^{-1}$ followed by $1000 \mathrm{~s}$ recovery at rest. The measuring time at each imposed strain rate or shear stress, determined from separate experiments to be adequate for achieving steady-state, was $54 \mathrm{~s}$ ( $24 \mathrm{~s}$ equilibration time, followed by $30 \mathrm{~s}$ measurement time).

The same pre-treatments were adopted for oscillatory shear experiments, which were conducted in two modes. For strainamplitude sweeps the measuring time at each value of the amplitude was $22 \mathrm{~s}$ (4 s equilibration, followed by $18 \mathrm{~s}$ measurement time) and the frequency was set at $1 \mathrm{~Hz}$. For the frequency-sweep experiments an optimum measuring time was selected for each frequency (increasing with decreasing frequency). and a constant strain amplitude of 0.05 (chosen to operate as far as possible within the linear viscoelastic regime) was applied. The frequency sweep was executed from high to low frequency in order to maximize the effect of the pre-treatment and therewith improve the reproducibility of the measurements. Total run times were of the order of 4 hours, consequently the low frequency points were determined when the sample had been gelling for a considerable time.

Creep measurements provide a link between low strain linear elastic behaviour and high strain steady shear flow, as well as giving time constants characteristic of the gel-network dynamics. Here a stress, $\tau_{0}$, was applied to the sample, and the strain response $\gamma$ was recorded as a function of time. After the stress had been applied for a time, $t_{1}$, chosen to ensure that the creep response had reached a steady state $(\mathrm{d} \dot{\gamma} / \mathrm{d} t=0$ or constant), it was removed and recovery of the strain $\gamma(t)$ was recorded. For these experiments the recovered gel pre-treatment protocol was used with a shorter rest recovery period of $200 \mathrm{~s}$.

\section{Results}

\section{Hectorite}

Oscillatory shear measurements. Fig. 3a shows the effect of increasing strain amplitude on the storage and loss modulus of

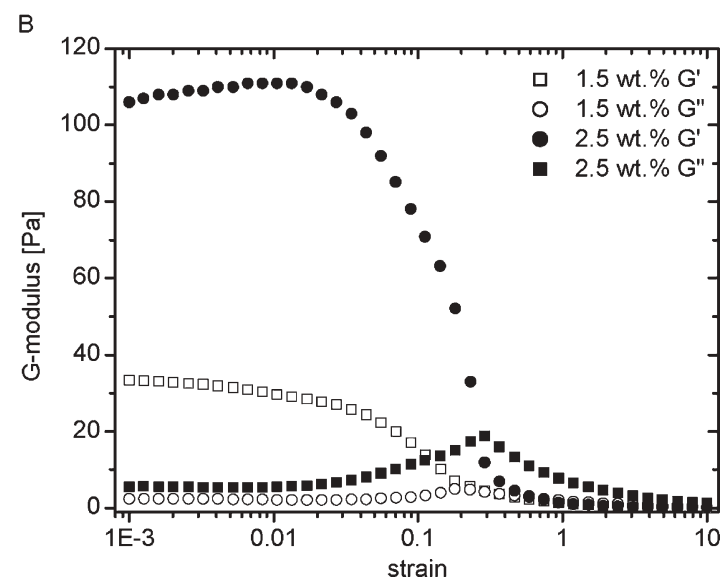

Fig. 3 Amplitude sweep for hectorite at two different concentrations. (a) broken gel, (b) recovered gel. 
the hectorite broken gel. For the $1.5 \mathrm{wt} \%$ fluid, $c / c^{*} \sim 1$; a low modulus gel is formed with $G^{\prime} \sim 25 \mathrm{~Pa}$ in the linear viscoelastic regime. The slow rise in $G^{\prime}$ (and fall of $G^{\prime \prime}$ ) with strain reflects the developing gelation of the 'broken gel' over the four minute period taken to reach a strain of 0.01 , below which negligible structure breakdown occurs. We have taken the peak value of $G^{\prime}$ in this low strain region, $G^{\prime}(\gamma \rightarrow 0)$, to characterise the strength of the undisturbed gel. Once the strain reaches $0.05, G^{\prime}$ starts to fall significantly and we can define this as an initial critical strain $\gamma_{\mathrm{c}}$ for structure breakdown and the limit of the linear regime. At the same time $G^{\prime \prime}$ rises and the curves cross as $G^{\prime \prime}$ goes through a maximum at $\gamma_{\mathrm{y}}$ $=0.25$; above this strain $G^{\prime}$ and $G^{\prime \prime}$ are similar and both low, the suspension behaving as a low viscosity liquid. $\gamma_{\mathrm{y}}$ is a less ambiguous measure of gel 'yield', and we term it the yield strain, though yielding clearly takes place over a range of strains and times and by $\gamma_{y}$ the material is already quite liquidlike. The values of $\gamma_{\mathrm{c}}$ and $\gamma_{\mathrm{y}}$, together with the corresponding values of $G^{\prime}$, enable us to estimate effective 'yield stress' values of the gel, $\tau_{\mathrm{c}}\left(=\gamma_{\mathrm{c}} G^{\prime}(\gamma \rightarrow 0)\right)$ and $\tau_{\mathrm{y}}\left(=\gamma_{\mathrm{y}} G_{\mathrm{y}}^{\prime}\right)$. These values are summarised in Table 3 .

For the $2.5 \mathrm{wt}^{\%} \%$ suspension, $c / c^{*} \sim 1.7$ and the gel is much stronger, with $G^{\prime}(\gamma \rightarrow 0) \sim 100 \mathrm{~Pa}$. Again the storage modulus falls and the loss modulus rises slightly at low strains, with initial yield taking place at $\gamma_{\mathrm{c}} \sim 0.01$ and the modulus curves crossing at $\gamma_{\mathrm{y}} \sim 0.3$ i.e. at strains very similar to the more dilute gel. Here $G^{\prime \prime}$ is significantly higher than $G^{\prime}$ above $\gamma_{\mathrm{y}}$ and the strain-induced gel-sol transition is clear.

Fig. 3b shows the amplitude-sweep behaviour of the corresponding hectorite recovered gels. At low strains, $G^{\prime}$ for both suspensions is about $10 \mathrm{~Pa}$ higher than for the broken gel, reflecting the extra $1000 \mathrm{~s}$ recovery time before measurements commenced. However, the values of $\gamma_{\mathrm{c}}$ and $\gamma_{\mathrm{y}}$ and the values of $G^{\prime}, G^{\prime \prime}$ in the non-linear yield regime are essentially the same for both pre-treatment procedures, indicating that strain is the critical parameter for structure breakdown, with only a weak dependence on the strength of the gel, and that once the gel starts to degrade the effects of previous flow history are rapidly erased.

We now examine the frequency dependence of the complex shear moduli in the linear regime as shown for the broken gels

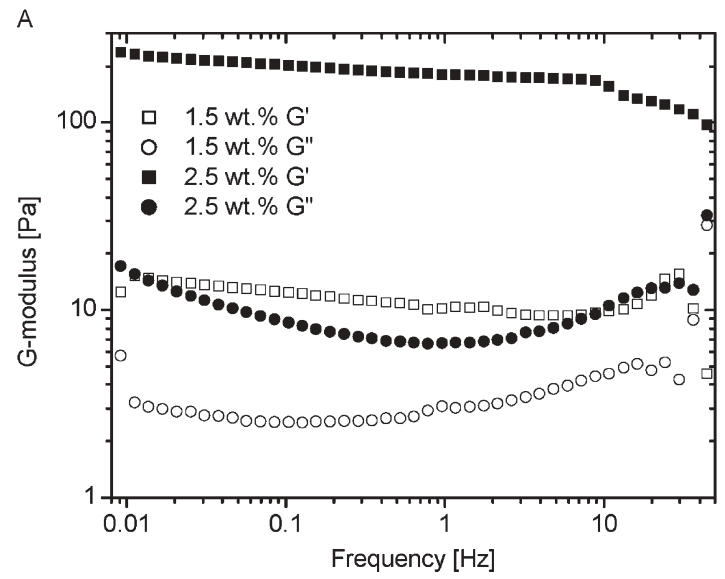

in Fig. 4a and for the recovered gels in Fig. 4b. The curves are rather structureless, with $G^{\prime}$ remaining essentially frequency independent for both suspensions and $G^{\prime \prime}$ about an order of magnitude lower until it starts to rise slowly above $3 \mathrm{~Hz}$ and rapidly above $30 \mathrm{~Hz}$, where the curves cross. The gradual rise in both moduli towards lower frequencies, especially for the broken gel, again reflects the developing gelation of the system over the measuring time, since measurements start at high frequency as explained in the Measurements section. The moduli at high frequency tend to be slightly lower than in the amplitude-sweep experiments for the same reason, but are consistent with them. Decreasing the strain amplitude from 0.05 to 0.005 increases the value of $G^{\prime}$ significantly, in line with Fig. 3. The values of $G^{\prime}$ at $1 \mathrm{~Hz}$ from these experiments are consistent with the values determined in the prior amplitudesweep study (see Table 3).

The kinetics of the build-up of the storage modulus for the $2.5 \mathrm{wt} \%$ hectorite gel for $\omega=0.5 \mathrm{~Hz}$ and $\gamma=0.05$ were also measured. It was observed that the gel reaches more than $50 \%$ of its limiting value in less than $30 \mathrm{~s}$ and approached a limiting value of about $110 \mathrm{~Pa}$ within ten minutes, although small further increases were seen for some hours. This is consistent with the values of $G^{\prime}$ and the small differences at small measuring times between the broken and recovered gels seen in Fig. 3 and 4. Similar timescales were found for the other suspensions studied in this work.

Creep experiments. This transition from gel to liquid-like behaviour is probed in more detail in the creep experiments, as shown in Fig. 5. The fluid shows an instantaneous elastic response, $\gamma_{\mathrm{e}}$, followed by a time-dependent creep region, leading finally to a linear flow region whose slope gives the (very low) shear rate corresponding to the imposed stress (the strain overshoot seen at small times and small applied stresses is an instrumental artefact arising from the finite inertia of the measurement assembly). The creep curves may be represented using a simple generalised Kelvin model: ${ }^{1}$

$$
\gamma(t)=\gamma_{e}+\sum_{\mathrm{i}} \bar{\gamma}_{\mathrm{i}}\left[1-e^{\frac{-t}{\lambda_{\mathrm{i}}}}\right]+\frac{t \tau}{\eta(\tau)}
$$

B



Fig. 4 Frequency sweep for hectorite at two different concentrations. (a) broken gel, (b) recovered gel. Strain amplitude $=0.05$. 


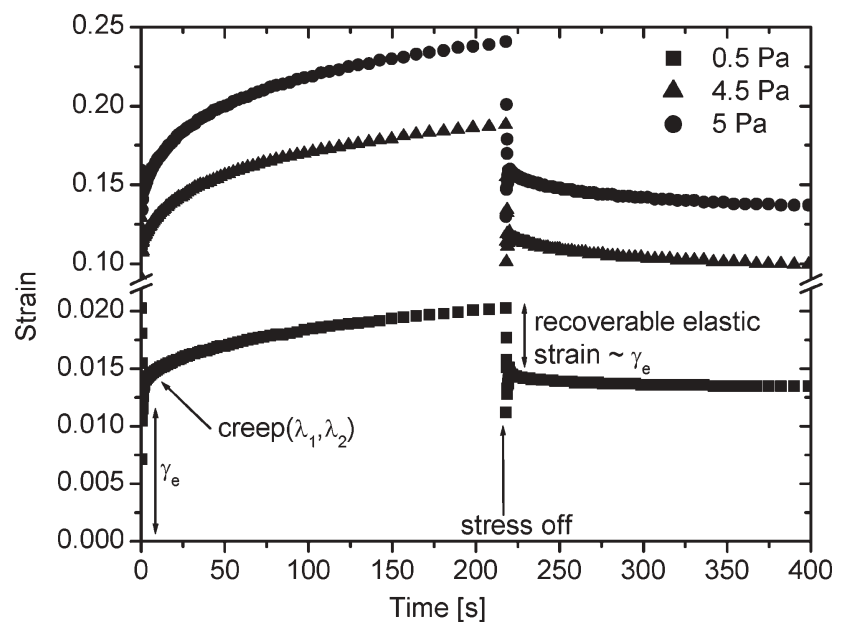

Fig. 5 Creep curves for Hectorite $2.5 \mathrm{wt} \%$; imposed stresses $0.5 \mathrm{~Pa}$, 4.5 $\mathrm{Pa}, 5 \mathrm{~Pa}$.

where $\lambda_{\mathrm{i}}$ are retardation times characterising the creep behaviour, $t$ is the applied stress, and $\eta(\tau)$ is the effective viscosity $=\tau / \dot{\gamma}$, where the shear-strain rate $\dot{\gamma}$ is simply the limiting slope of the linear flow region. Two retardation times were found to be sufficient to describe the time-dependent creep of these fluids. A summary of the parameters corresponding to the curves is given in Table 2. Eqn (2) is an excellent representation of the data, with regression coefficients $R^{2}$ typically greater than 0.95 , except for the lowest applied stresses $(\tau<\sim 2 \mathrm{~Pa}$ ) where the measured strains are very small and $R^{2}$ dropped as low as 0.75 . It can be seen that extremely low shear rates are observed for the initial stages of flow, $<10^{-5} \mathrm{~s}^{-1}$, much lower than can be achieved in imposed shearrate experiments (see below). Above $8 \mathrm{~Pa}$, there is a sudden and dramatic increase in the observed shear rate, by about four orders of magnitude. Here flow dominates and the elastic/creep parameters are not measurable. The initial low-time elastic response of the gel defines a creep compliance $J_{\mathrm{e}}(t \rightarrow 0)$ or plateau modulus $(\omega \rightarrow \infty) G_{\mathrm{e}}=\tau / \gamma_{\mathrm{e}}$. These values are also given in Table 2. The two retardation times $\lambda_{1}$ and $\lambda_{2}$ are about $100 \mathrm{~s}$ and $10 \mathrm{~s}$, respectively, and go through a maximum at an imposed stress of $2 \mathrm{~Pa}$ (see Fig. 6). The amplitude of the slower time, $\gamma_{1}$, is 2-3 times that of $\lambda_{2}\left(\gamma_{2}\right)$ for all applied stresses up to $8 \mathrm{~Pa}$. The postcreep viscosity, $\eta(\tau)$, is plotted in Fig. 7. This clearly demonstrates that these systems have a high but finite viscosity of $2-10 \times 10^{4} \mathrm{~Pa} \mathrm{~s}$ at low stresses before falling by six orders of magnitude over a small range of stress around $8.5 \mathrm{~Pa}$, which we may identify with the effective yield stress of this fluid - the stress at which the material transforms from an elastoviscous gel with a high modulus ( $\sim 30-50 \mathrm{~Pa})$ and very high viscosity to a low viscosity liquid, which continues to shear thin from a post-yield value of $24 \mathrm{mPas}$. The strain at which this dramatic transformation occurs is 0.25 , consistent with the values of $\gamma_{y}$ observed in the amplitude sweep oscillatory shear experiments. The values of $G_{\mathrm{e}}$ from these creep experiments are also in line with those found for $G^{\prime}$ in the oscillatory measurements (see Table 3 at the end of this section).

On removing the stress from the samples after a steady flow regime has been achieved $(\sim 200 \mathrm{~s})$, the strain initially falls rapidly in a few seconds to give a recoverable elastic strain $\gamma_{r}^{\mathrm{i}}$, followed by a long time creep recovery $\gamma_{r}^{\mathrm{cr}}$. The values of $\gamma_{\mathrm{r}}^{\mathrm{i}}$, however, are only about $50 \%$ of the corresponding $\gamma_{\mathrm{e}}$ values, this ratio tending to decrease with increasing applied stress. Similarly, the amplitude of recovered creep strain is only $\sim 40 \%$ of that observed during the stress-application stage, this ratio again falling at higher stresses. These observations indicate that the coherence of the structure responsible for the sample's elastic behaviour has been significantly decreased during the 200 seconds of stress application. These data show that whilst the system still retains its very high viscosity (up to applied stresses of $8 \mathrm{~Pa}$ ), its elastic behaviour is seriously compromised by extended periods of stress application - prolonged application of stresses well below the 'yield stress' at which viscosity falls dramatically cause the gel to become elastically challenged.

Continuous steady shear measurements. Finally we move to the steady shear experiments for which the shear stresscontrolled shear-rate flow curves are displayed in Fig. 8a for the 1.5 and $2.5 \mathrm{wt} \%$ broken gels. As the imposed shear rate is increased for the $1.5 \mathrm{wt} \%$ suspension, the shear stress initially increases until $\dot{\gamma} \sim 0.1 \mathrm{~s}^{-1}$, whereafter it decreases before going through a minimum at $\dot{\gamma} \sim 5 \mathrm{~s}^{-1}$. It continues to increase rapidly with shear rate until $\dot{\gamma} \sim 10 \mathrm{~s}^{-1}$, when there is a sharp break in slope as the rate of shear-stress growth decreases dramatically before building steadily at higher shear rates. The flow curve is therefore highly non-monotonic, displaying extrema at $\dot{\gamma} \sim 0.1$ and $10 \mathrm{~s}^{-1}$, showing similar behaviour to that recently observed for

Table 2 Kelvin model parameters for creep curves of hectorite $2.5 \mathrm{wt} \%$ gel

\begin{tabular}{|c|c|c|c|c|c|c|c|c|c|c|}
\hline$\tau / \mathrm{Pa}$ & $\gamma_{\mathrm{e}}$ & $G_{\mathrm{e}} / \mathrm{Pa}$ & $\gamma_{1}$ & $\gamma_{2}$ & $\lambda_{1} / \mathrm{s}$ & $\lambda_{2} / \mathrm{s}$ & $\dot{\gamma} / \mathrm{s}^{-1}$ & $\eta(\tau) / \mathrm{Pa} \mathrm{s}$ & $\gamma^{\mathrm{i}}$ & $\gamma_{r}^{\mathrm{cr}}$ \\
\hline 0.05 & 0.0059 & 8.5 & 0.0022 & 0.0011 & 87.9 & 5.8 & $3.0 \mathrm{E}-06$ & 16617 & - & - \\
\hline 0.1 & 0.0074 & 13.5 & 0.0022 & 0.0009 & 63.5 & 7.1 & $5.6 \mathrm{E}-06$ & 17776 & - & - \\
\hline 0.5 & 0.0130 & 38.5 & 0.0035 & 0.0017 & 65.6 & 5.3 & $1.1 \mathrm{E}-05$ & 47100 & 0.0064 & 0.0066 \\
\hline 1 & 0.0218 & 45.9 & 0.0057 & 0.0018 & 69.9 & 7.3 & $1.7 \mathrm{E}-05$ & 60534 & 0.0122 & 0.0149 \\
\hline 2 & 0.0409 & 48.9 & 0.0131 & 0.0045 & 104.2 & 17.1 & $1.9 \mathrm{E}-05$ & 105620 & 0.0249 & 0.0331 \\
\hline 3 & 0.0584 & 51.4 & 0.0178 & 0.0061 & 71.7 & 9.8 & $4.5 E-05$ & 66527 & 0.0350 & 0.0484 \\
\hline 4 & 0.0874 & 45.8 & 0.0283 & 0.0099 & 48.1 & 5.7 & $9.5 E-05$ & 42054 & 0.0541 & 0.0729 \\
\hline 4.5 & 0.1093 & 41.2 & 0.0392 & 0.0117 & 38.3 & 3.7 & $1.3 \mathrm{E}-04$ & 34198 & - & - \\
\hline 5 & 0.1344 & 37.2 & 0.0523 & 0.0167 & 33.2 & 2.5 & $1.8 \mathrm{E}-04$ & 28287 & 0.0790 & 0.1020 \\
\hline 5.5 & 0.1612 & 34.1 & 0.0723 & 0.0266 & 28.6 & 1.7 & $2.3 E-04$ & 23500 & - & - \\
\hline 6 & 0.1866 & 32.2 & 0.0937 & 0.0401 & 22.8 & 1.1 & $2.9 \mathrm{E}-04$ & 20777 & 0.0870 & 0.1220 \\
\hline 7 & 0.2233 & 31.3 & 0.1835 & 0.1100 & 15.1 & 0.5 & $4.9 \mathrm{E}-04$ & 14397 & 0.0880 & 0.1430 \\
\hline 8 & 0.2523 & 31.7 & 0.5696 & 0.2415 & 10.7 & 0.4 & $8.7 \mathrm{E}-04$ & 9165 & - & - \\
\hline 9 & - & - & - & - & - & - & $3.8 \mathrm{E}+02$ & 0.024 & - & - \\
\hline 10 & - & - & - & - & - & - & $6.3 \mathrm{E}+02$ & 0.016 & - & - \\
\hline
\end{tabular}




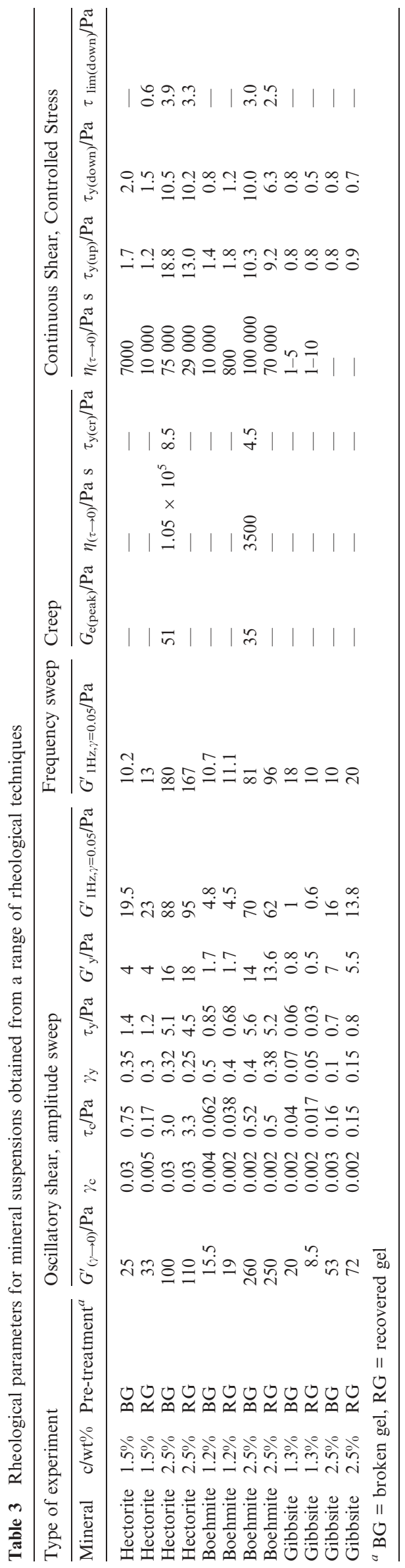

surfactant fluids and other shear-banding systems. ${ }^{37,38}$ The $2.5 \mathrm{wt} \%$ suspension shows similar behaviour with the extrema displaced very slightly to higher shear rates.

After the highest shear rate $\left(500 \mathrm{~s}^{-1}\right)$ has been applied and the applied shear rate is decreased, the $1.5 \mathrm{wt} \%$ fluid follows the 'up' curve almost exactly, down as far as the abrupt slope change at $\dot{\gamma} \sim 10 \mathrm{~s}^{-1}$. However, the 'down' curve exhibits a shallow minimum near this point and instead of the shear stress dropping sharply, it rises smoothly until $\dot{\gamma} \sim 3 \mathrm{~s}^{-1}$, whereupon it starts to decrease again. It continues to do so until $\dot{\gamma} \sim 0.1 \mathrm{~s}^{-1}$, whereafter it rises gently and appears to be approaching a limiting shear stress of $\sim 0.55 \mathrm{~Pa}$ at very low shear rates. So although the 'down' curves starts off coincident with the 'up' curve, the fluid is re-gelling under a very different shear history than it experienced on the 'up' curve; this is reflected in major differences in its flow curve. Again, the $2.5 \mathrm{wt} \%$ fluid shows similar behaviour with its 'down' flow curve.

Let us now take the flow curves displayed in Fig. 8a, and consider how the fluid should respond in a controlled shearstress experiment. For the $1.5 \mathrm{wt} \%$ fluid the shear rate will increase as shear stress is raised until a stress of $\sim 2 \mathrm{~Pa}$ at $\dot{\gamma} \sim 0.1 \mathrm{~s}^{-1}$. Here the slope of the flow curve becomes negative, representing an unstable flow regime, and the shear rate should jump to a value corresponding to $\tau=2 \mathrm{~Pa}$ on the upper positive slope branch i.e. $\dot{\gamma} \sim 100 \mathrm{~s}^{-1}$. Fig. $8 \mathrm{~b}$ shows that this is precisely what does occur, with an abrupt jump in shear rate at a shear stress of $\sim 2 \mathrm{~Pa}$. This flow curve reaches much lower shear rates and stresses than Fig. 8a, and shows signs of shear thickening at the lowest stresses. The $1.5 \mathrm{wt} \%$ down curve in Fig. 8a also implies an abrupt jump to lower shear rates once the stress drops to the shallow minimum at $\sim 1 \mathrm{~Pa}, \dot{\gamma} \sim 20 \mathrm{~s}^{-1}$. Again, this is observed in the controlled stress experiment in Fig. 8b. Similar abrupt jumps in shear rate are observed for the $2.5 \mathrm{wt} \%$ fluid in Fig. $8 \mathrm{~b}$, at stresses and strain rates consistent with the non-monotonic flow curves of Fig. 8a. Note the complementary abrupt change in shear stress from $\sim 10 \mathrm{~Pa}$ to $\sim 4 \mathrm{~Pa}$ as the shear rate remains at $\sim 30 \mathrm{~s}^{-1}$. This is qualitatively consistent with the rapid but not quite so abrupt changes in shear stress observed at slightly lower shear rates in Fig. 8a. Since it is difficult to reproduce exactly the same flow curves for these highly shear-sensitive fluids even when they experience as near the same shear history as possible, the consistency between the controlled shear rate and controlled shear-stress flow curves is impressive (see also Da Cruz et al. ${ }^{39}$ ).

Fig. 8c shows the viscosity-shear stress plot corresponding to Fig. $8 \mathrm{~b}$ for the fluids with the 'broken gel' pre-treatment protocol. The $1.5 \mathrm{wt} \%$ fluid shows the low shear-stress thickening behaviour anticipated in Fig. 8b. This may simply be due to structure rebuilding in the fluid after its pre-shearing phase or it could indicate shear-induced gelation at these low shear stresses. The fluid effective viscosity remains constant at the high value of $10^{4} \mathrm{~Pa} \mathrm{~s}$ before starting to shear-thin gradually, until the abrupt reduction in viscosity at $\tau \sim 1.8 \mathrm{~Pa}$ arising from the large shear-rate reduction experienced here. Once the viscosity falls to about $10 \mathrm{mPa}$, the viscosity continues to fall gently, in a power-law manner, as observed for many clay fluids usually described as 'viscoplastic' e.g. those described by a Herschel-Bulkley model, where powerlaw flow follows once the fluid starts to flow at the so-called 'yield stress' $\tau_{\mathrm{y}}$, below which the fluid is assumed not to flow. It 

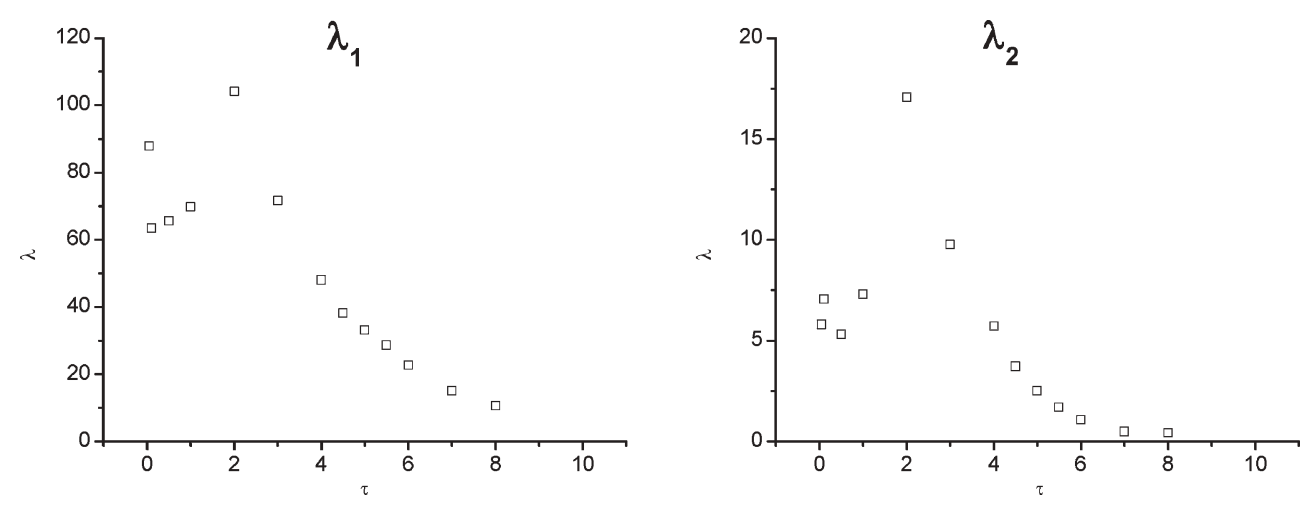

Fig. 6 Retardation times $\lambda$ (s) for Hectorite $2.5 \mathrm{wt} \%$ as a function of the applied stress (Pa).

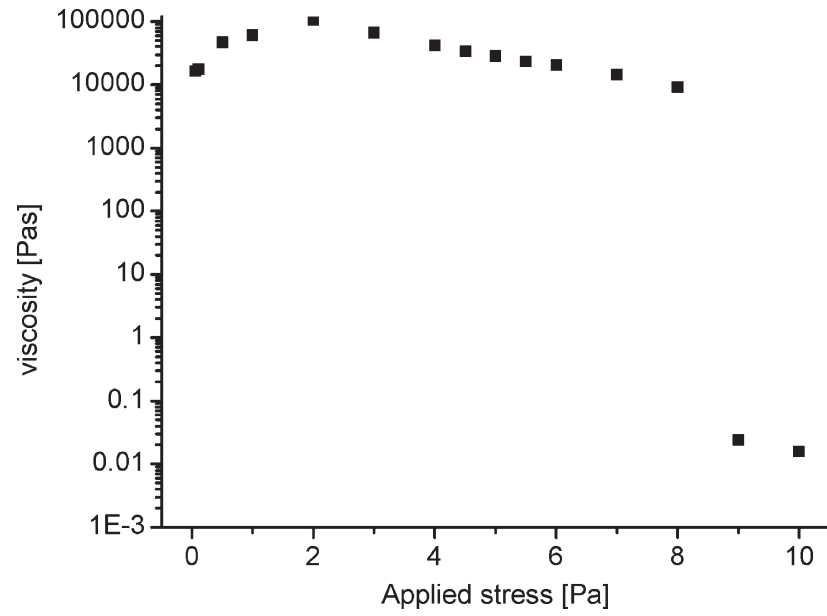

Fig. 7 Post-creep viscosity as a function of applied stress for hectorite $2.5 \mathrm{wt} \%$.

can be seen here that the sharp drop in stress at $\tau_{\mathrm{y}} \sim 1.8 \mathrm{~Pa}$ characterises such 'yield' behaviour but that the material continues to flow below this stress, albeit with a viscosity several (here six) orders of magnitude higher than the commonly observed fluid viscosity. The re-healing 'down' fluid eventually shows a higher viscosity than the 'up' fluid before gelling at a slightly higher shear stress $(\sim 2 \mathrm{~Pa})$, and continuing to increase in viscosity as lower and lower stresses are applied. For the $2.5 \mathrm{wt} \%$ fluid, similar behaviour is observed as the applied shear stress increases, with the low stress viscosity plateauing at $\sim 10^{5} \mathrm{~Pa}$ s and an effective yield stress $\sim 20 \mathrm{~Pa}$, both values being consistent with the creep experiments for this fluid (compare Fig. 7 and 8c, which are remarkably similar). The re-healing fluid starts to gel at $\tau \sim 10 \mathrm{~Pa}$ before the gel anneals at constant shear rate and increases rapidly towards a viscosity of $>10^{6} \mathrm{~Pa}$ s over a short range of stress from 4 to $1.5 \mathrm{~Pa}$. We will return to this feature in the Discussion.

Fig. 9a,b,c show similar behaviour for the fluids having the 'recovered gel' pre-treatment protocol. This similarity indicates that for these hectorite fluids, the re-building of microstructure is sufficiently rapid that the additional $1000 \mathrm{~s}$ of rest experienced by the 'recovered' gels only has a minor influence on the results. The same phenomena are observed, at essentially the same shear stresses and with consistent viscosity values at both high and low applied stresses. The annealing transition for the re-healing fluid at constant shear rate is perhaps more pronounced for these fluids - see Fig. 9b.

Hence different flow curves are obtained for controlled stress and controlled strain-rate experiments on the same fluid; multiple repeat experiments on both fresh and re-cycled samples confirm the reproducibility to within about $10 \%$ in the measured variable of the curves typified by Fig. 8 and 9 . We shall see later that the same generic behaviour is observed for all the systems studied. Whilst the results for the apparent viscosity and bulk-gel yield values from these experiments are consistent with those from the transient creep and oscillatory measurements, the results indicate the care that is needed in the interpretation of high strain continuous shear experiments for particulate-gelling fluids of this type.

Table 3 summarises the rheological parameters of the two hectorite suspensions studied here by a range of rheological techniques. Similar values of the same property, at similar stress-strain conditions, are obtained from the oscillatory, creep and continuous shear flow experiments. A consistent picture emerges of a weakly gelling fluid at $c / c^{*} \sim 1$, having a plateau modulus $G_{\infty}$ of $30 \mathrm{~Pa}$ and an effective viscosity of $\sim 10^{4} \mathrm{~Pa} \mathrm{~s}$ at low stresses, transforming at a critical 'yield' or transition stress of 1.0-1.5 Pa into a shear-thinning suspension of high stress viscosity about $10 \mathrm{mPa} \mathrm{s}$ and a power-law exponent $\sim 0.5$. For the more concentrated system at $c / c^{*}$ $\sim 1.7$, the same qualitative behaviour is seen but with much higher values of the low stress plateau modulus (100 Pa) and effective viscosity $\left(10^{5} \mathrm{~Pa} \mathrm{~s}\right)$ and of the transition stress (10 Pa). However, the transition or 'yield' strains for both fluids are similar, $\sim 0.05$ for initial onset and $\sim 0.25$ for dramatic modification of the clay network and destruction of its continuous gel characteristics.

\section{Boehmite}

Fig. 10a displays the amplitude-sweep oscillatory shear data for boehmite suspensions, and we see immediately that these are qualitatively different from those exhibited by hectorite in Fig. 3. For the $1.2 \mathrm{wt} \%$ suspension $\left(c / c^{*} \sim 1.1\right)$ both $G^{\prime}$ and $G^{\prime \prime}$ are very low. The limiting low strain modulus $G^{\prime}(\omega=1 \mathrm{~Hz})=$ $30 \mathrm{~Pa}$, a similar value to that for hectorite at a similar reduced concentration $c / c^{*} \sim 1.0$. However $G^{\prime}(\omega=1 \mathrm{~Hz})$ falls away from its limiting value at $\gamma_{\mathrm{c}} \sim 0.003$, an order of magnitude 
A

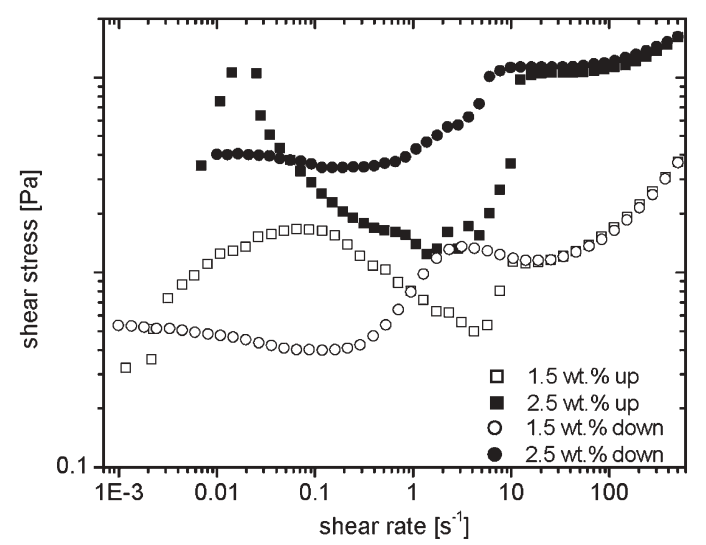

C

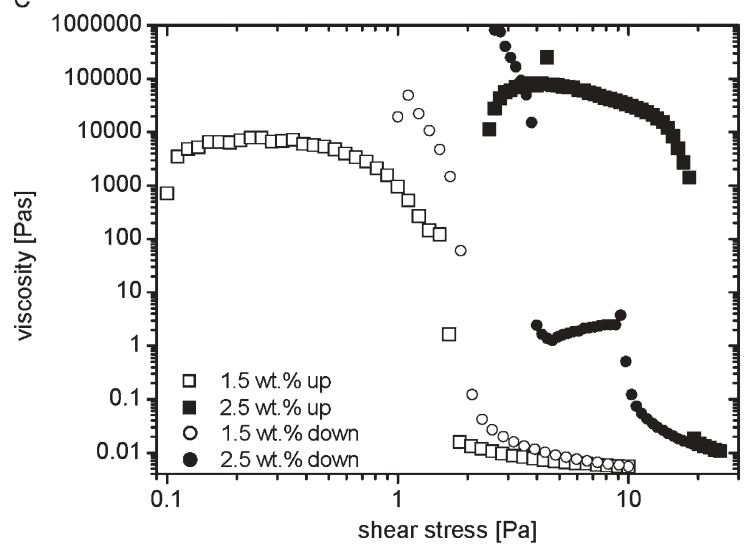

B

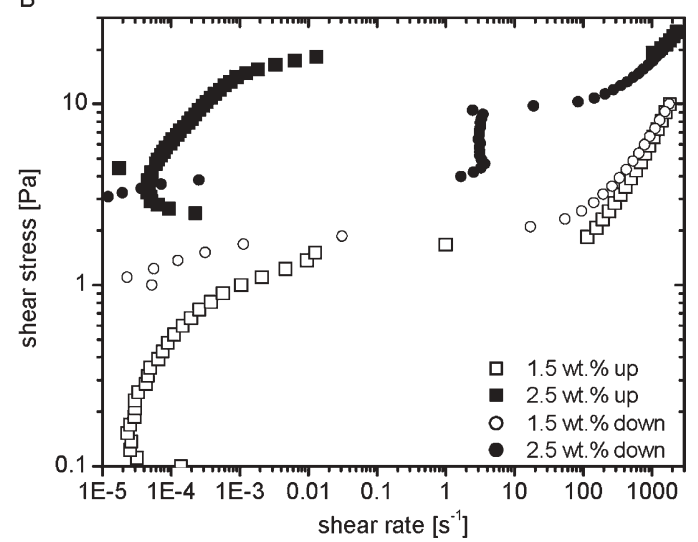

Fig. 8 Flow curves for hectorite at two different concentrations for broken gels: (a) shear stress-controlled shear rate, (b) controlled shear stressshear rate, (c) viscosity-controlled shear stress.

smaller than for hectorite. $G^{\prime \prime}$ displays a weak maximum at $\gamma \sim 0.5$, where it becomes comparable with $G^{\prime}$. For the higher concentration $2.5 \mathrm{wt} \%$ suspension, having $c / c^{*} \sim 2.3$, the moduli are much higher and display a more complex strain dependence: $G^{\prime}(\gamma \rightarrow 0) \sim 250 \mathrm{~Pa}$, with an indication that $\gamma_{\mathrm{c}} \sim 0.002$ as before but with $G^{\prime}$ falling away rapidly with increasing strain. An intermediate inflexion is observed between $\gamma \sim 0.01$ and 0.1 , whereafter $G^{\prime}$ falls to values comparable with those for the more dilute suspension at high strains. The loss modulus also falls rapidly with strain before exhibiting a weak maximum and crossing the $G^{\prime}$ curve at $\gamma_{\mathrm{y}} \sim 0.4$, a value very similar to that for the lower concentration suspension and for the hectorite suspensions.

The recovered boehmite gels showed very similar behaviour (Fig. 10b), except that the slope change near $\gamma \sim 0.01$ is more marked. This indicates that boehmite gels somewhat quicker than hectorite and that the 'broken gel' protocol is sufficient to approach a steady-state gel.

The frequency sweep experiments were carried out at $\gamma \sim 0.05$, on the intermediate plateau or inflexion region of Fig. 10a,b. As seen in Fig. 11, both $G^{\prime}$ and $G^{\prime \prime}$ are relatively constant with frequency over the range studied, typical of significantly gelled systems. The values are consistent with Fig. 10a,b at this imposed strain, and the slight increases with frequency mainly reflect the increasing gelation of the samples over the several hours taken to acquire the data, from low to high frequencies. As expected from above, the pre-measurement protocols had little influence on the results.

The $2.5 \mathrm{wt} \%$ suspension showed creep behaviour similar to that of hectorite in Fig. 5. The Kelvin model parameters are given in Table 4. The data show the same features as hectorite but reflect a slightly weaker gel at a somewhat higher value of $c / c^{*}$. The peak instantaneous modulus $G_{\mathrm{e}}$ is $35 \mathrm{~Pa}$, about $60 \%$ of $G^{\prime}(1 \mathrm{~Hz})$ as found for hectorite. The data are again fitted by two retardation times, of order $50 \mathrm{~s}$ and $5 \mathrm{~s}$, with the longer time accounting for about $75 \%$ of the response, again in line with hectorite. However, the low shear viscosity is about an order of magnitude lower than for the hectorite gel, and drops abruptly at an applied stress of $4.5 \mathrm{~Pa}$, the apparent yield stress above which the fluid flows with a viscosity lower by more than three orders of magnitude (see Fig. 12). The critical strain for the transition is $\sim 0.15$.

The controlled shear-rate flow curve is displayed in Fig. 13a. For $c / c^{*} \sim 1.1$, the 'up' flow curve is similar to those observed for hectorite, with extrema in shear stress occurring at 1 and $7 \mathrm{~s}^{-1}$. This is reflected in the sharp increase in shear rate observed at a stress of $\sim 1.3 \mathrm{~Pa}$ in the controlled stress experiment, Fig. 13b, and in the rapid decrease in viscosity at this 'yield' stress in Fig. 13c. The re-healing 'down' curve in Fig. 13a remains at essentially constant stress after the 'down' flow curve separates from the 'up' curve below $\sim 20 \mathrm{~s}^{-1}$. The controlled stress re-healing curve, on the other hand, appears to follow the 'down' trajectory expected if the fluid re-tracked 

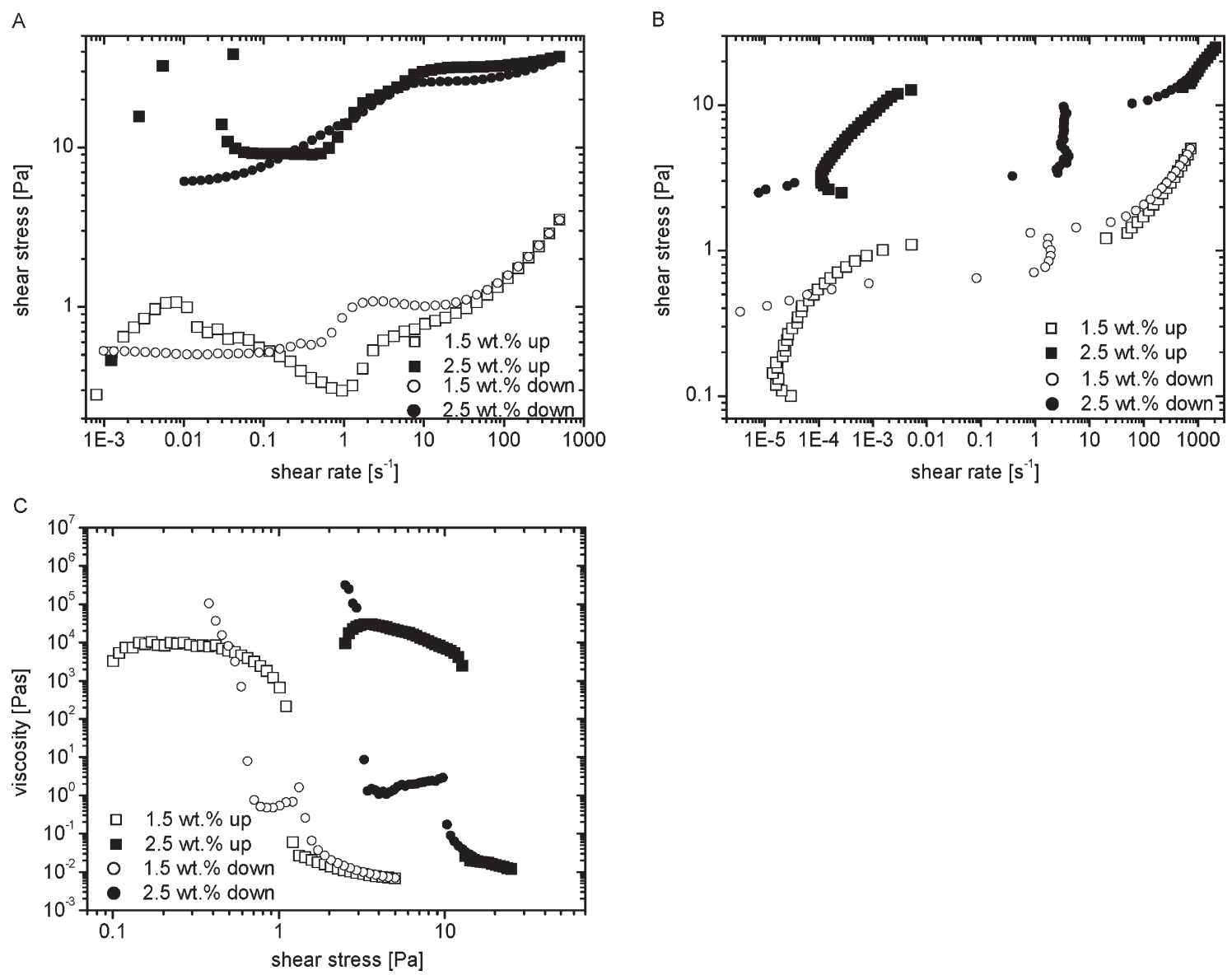

Fig. 9 Flow curves for hectorite at two different concentrations for recovered gels: (a) shear stress-controlled shear rate, (b) controlled shear stress-shear rate, (c) viscosity-controlled shear stress.

the 'up' curve in Fig. 13a, showing an abrupt transition at a stress of $\sim 0.9 \mathrm{~Pa}$, from $\dot{\gamma} \sim 10 \mathrm{~s}^{-1}$ to $\dot{\gamma} \sim 0.03 \mathrm{~s}^{-1}$. As the stress is decreased further the shear rate remains essentially constant, reflecting the constant viscosity regime (see Fig. 13c) also observed for the hectorite gels.

By contrast, for the $2.5 \mathrm{wt} \%$ curve $\left(c / c^{*} \sim 2.3\right)$ the flow curves for the 'up' and 'down' cycles in Fig. 13a are relatively flat, structureless and similar. The controlled stress flow curves in Fig. 13b are similar to the 'up' curve for the

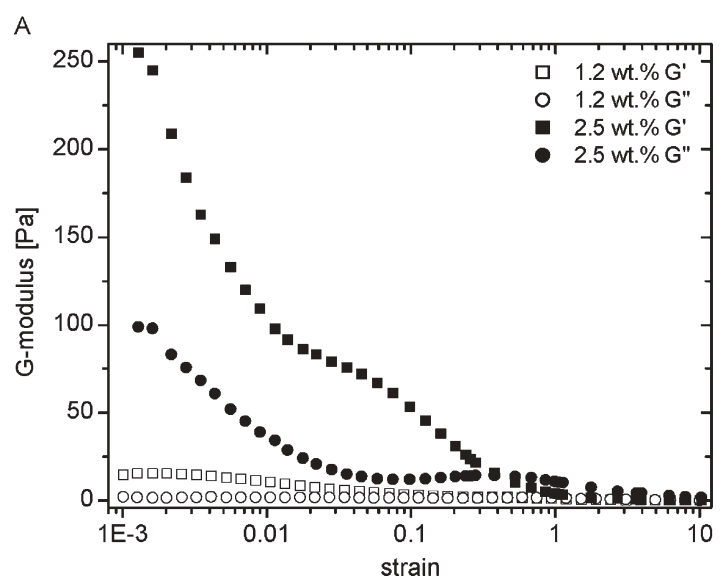

$1.2 \mathrm{wt} \%$ fluid, displaced to higher stresses and lower shear rates. This suggests a shallow maximum-minimum in the flow curve at shear rates well below those accessible in Fig. 13a. This is reflected in Fig. 13c, where a low stress viscosity of $\sim 10^{5} \mathrm{~Pa} \mathrm{~s}$ falls dramatically at a 'yield' stress of $10 \mathrm{~Pa}$ to the high stress, low viscosity-flow regime. The behaviour of the recovered gel pre-treatment fluids is essentially the same (see Fig. 13d), as expected of this relatively rapidly gelling system.

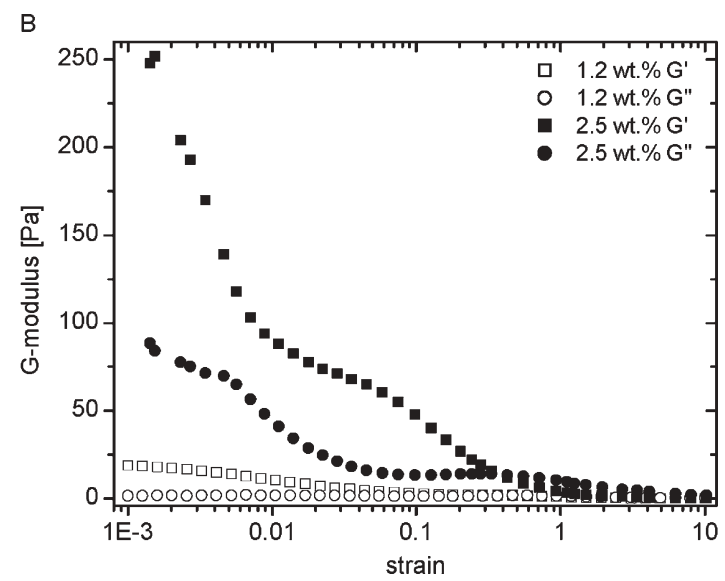

Fig. 10 Amplitude sweep for boehmite at two different concentrations. (a) Broken gel, (b) recovered gel. 


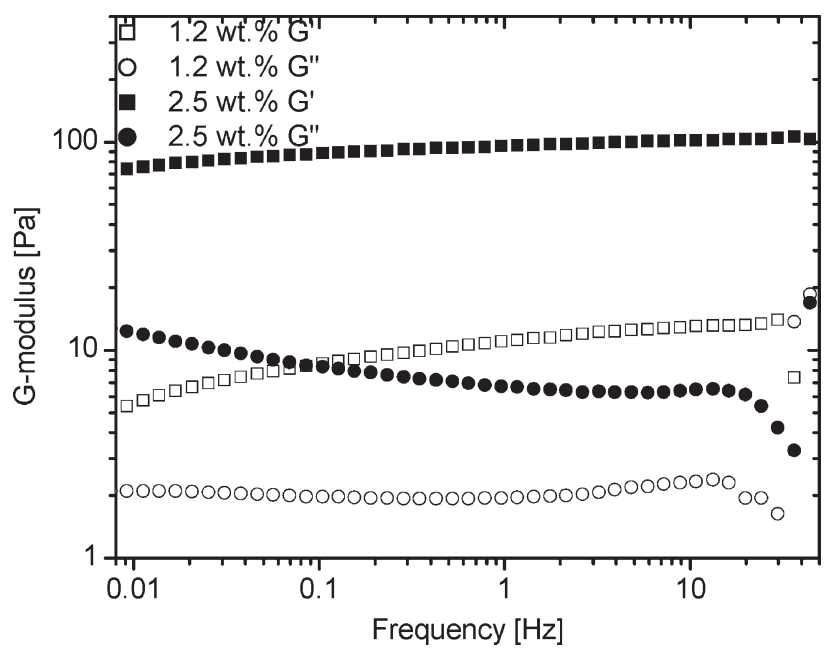

Fig. 11 Frequency sweep for boehmite at two different concentrations for recovered gel.

Table 4 Kelvin model parameters for creep curves of boehmite $2.5 \mathrm{wt} \%$

\begin{tabular}{llllllllr}
\hline$\tau / \mathrm{Pa}$ & $\gamma_{\mathrm{e}}$ & $G_{\mathrm{e}} / \mathrm{Pa}$ & $\gamma_{1}$ & $\gamma_{2}$ & $\lambda_{1} / \mathrm{s}$ & $\lambda_{2} / \mathrm{s}$ & $\dot{\gamma} / \mathrm{s}^{-1}$ & $\begin{array}{r}\eta(\tau) / \\
\mathrm{Pa} \mathrm{s}\end{array}$ \\
\hline 0.01 & 0.0410 & 0.2 & 0.0247 & 0.0100 & 72.1 & 8.2 & $6.2 \mathrm{E}-05$ & 162 \\
0.05 & 0.0418 & 1.2 & 0.0241 & 0.0082 & 45.5 & 4.5 & $9.7 \mathrm{E}-05$ & 516 \\
0.1 & 0.0398 & 2.5 & 0.0222 & 0.0071 & 39.9 & 3.4 & $1.1 \mathrm{E}-04$ & 907 \\
0.5 & 0.0486 & 10.3 & 0.0375 & 0.0119 & 47.7 & 4.5 & $1.5 \mathrm{E}-04$ & 3305 \\
1 & 0.0644 & 15.5 & 0.0651 & 0.0191 & 47.4 & 4.4 & $2.7 \mathrm{E}-04$ & 3654 \\
1.5 & 0.0812 & 18.5 & 0.1170 & 0.0307 & 44.8 & 4.2 & $4.6 \mathrm{E}-04$ & 3236 \\
2 & 0.0983 & 20.3 & 0.1763 & 0.0480 & 44.3 & 5.1 & $7.0 \mathrm{E}-04$ & 2841 \\
2.5 & 0.1179 & 21.2 & 0.2665 & 0.0699 & 41.7 & 4.7 & $1.2 \mathrm{E}-03$ & 2129 \\
2.75 & 0.0785 & 35.0 & 0.1573 & 0.0447 & 45.3 & 6.5 & $8.7 \mathrm{E}-04$ & 3145 \\
3 & 0.1377 & 21.8 & 0.3245 & 0.1081 & 37.1 & 4.6 & $1.7 \mathrm{E}-03$ & 1721 \\
3.5 & 0.1364 & 25.7 & 0.3128 & 0.1216 & 35.5 & 4.7 & $2.4 \mathrm{E}-03$ & 1449 \\
3.75 & 0.1752 & 21.4 & 0.4256 & 0.1553 & 15.0 & 1.5 & $9.3 \mathrm{E}-03$ & 404 \\
4 & 0 & - & 0.4677 & 0.2715 & 6.1 & 0.1 & $1.5 \mathrm{E}-02$ & 274 \\
5 & - & - & - & - & - & - & $8.5 \mathrm{E}-01$ & 5.9 \\
6 & - & - & - & - & - & - & $3.5 \mathrm{E}+00$ & 1.7 \\
\hline
\end{tabular}

The rheological parameters determined by the different techniques are summarised in Table 3. The picture that emerges for boehmite is that in many ways it forms gels similar in strength at comparable $c / c^{*}$ with hectorite. It gels

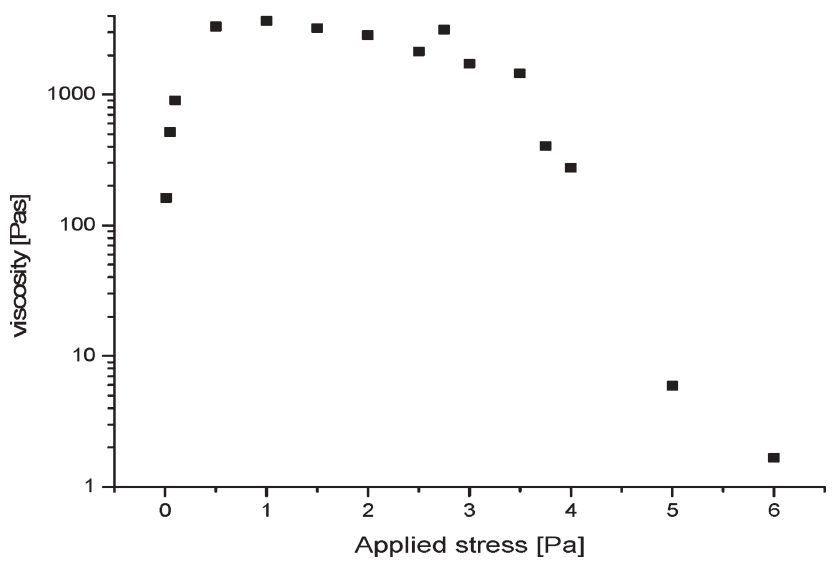

Fig. 12 Post-creep viscosity as a function of applied stress for boehmite $2.5 \mathrm{wt} \%$. and degels somewhat more quickly than hectorite, probably reflecting the faster Brownian rotation of the rods compared to the lath-like hectorite. The boehmite gels start to fail at significantly lower strains than for hectorite, though the strain $\gamma_{\mathrm{y}}$ for major failure is comparable but the gel-sol transition is less abrupt.

\section{Gibbsite}

The two gibbsite suspensions of concentration $1.3 \mathrm{wt} \%$ and $2.5 \mathrm{wt} \%$ correspond to $c / c^{*} \sim 0.05$ and 0.09 , respectively, much lower than for the hectorite and boehmite systems studied above. The oscillatory shear amplitude sweep data in Fig. 14 behave in a similar manner to those for boehmite (Fig. 10); the low strain values of $G^{\prime}$ are 5 and $70 \mathrm{~Pa}$, respectively, reflecting the very weakly gelled nature of these lower $c / c^{*}$ systems. The crossover strain is $\gamma_{\mathrm{y}} \sim 0.1$; this is lower but similar to that for the other two particle types. Again there is only a small difference between the broken and recovered gel pre-conditioning protocols. The storage and loss moduli show the essentially constant values typical of a gelled network over the wide frequency range 0.1 to $20 \mathrm{~Hz}$ (see Fig. 15) with values consistent with the strain used, $\gamma=0.05$, which for this system is well into the non-linear region. $G^{\prime}$ is just slightly larger than $G^{\prime \prime}$ in both cases, indicative of very weak gels. The flow curves for the gibbsite fluids are relatively structureless compared to the other fluids (see Fig. 16a, 16b for the broken gel pre-treatment). Fig. 16c shows that for both pre-treatment protocols, the low stress viscosities are 1-5 $\mathrm{Pa} s$ (though wall slip may be present in this region), with yield stresses $\tau \sim 0.7 \mathrm{~Pa}$, largely independent of concentration, in contrast to the hectorite and boehmite systems which transcend the $c<c^{*}$ to $c>c^{*}$ regions.

Because the gibbsite suspensions have $c \ll c^{*}$, they behave as typical viscoplastic fluids/weak gels with $G^{\prime}$, effective viscosities and yield stresses all much lower than the hectorite and boehmite suspensions of similar mass concentration but very different effective volume fraction as measured by $c / c^{*}$. Qualitatively however, they show similar rheological behaviour to boehmite, especially in the high strain-dependence of $G^{\prime}$ and $G^{\prime \prime}$.

\section{Discussion}

The results represent an attempt to give a comprehensive characterisation of the rheology of a series of anisotropic colloidal suspensions, commonly described as 'viscoplastic', as they make the transition from an essentially elastic solid to a low viscosity liquid on being subjected to increasing shear stress or strain. In order to do this, we have adopted an approach with two key elements. The first is careful pretreatment of the fluid before measurements are made. By adopting protocols that essentially destroy the particlemicrostructural network by application of high and prolonged shear flow, and then allowing sufficient time for this to re-form into an essentially fully restructured gel, it is possible to make reproducible measurements and to characterise the properties of a soft material in a well-defined initial state (as a guideline, a suitable protocol uses a recovery time which is at least 10 times longer than the $80 \%$ recovery time of $\left.G^{\prime}(\omega=1 \mathrm{~Hz})\right)$. The 
A

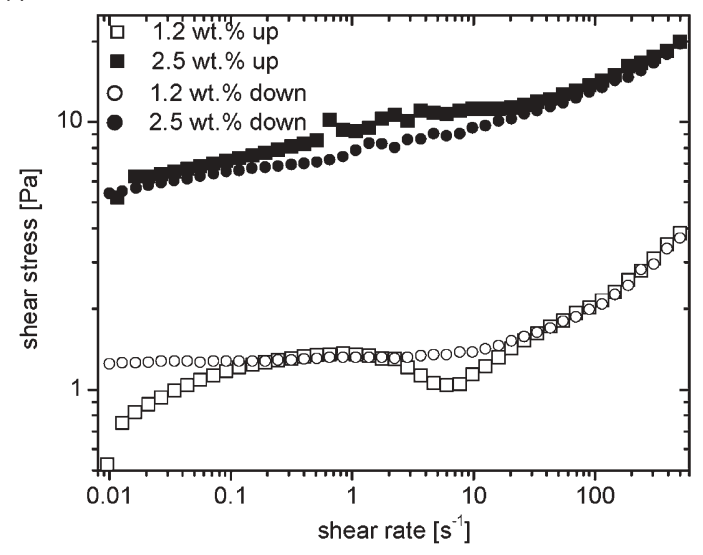

C

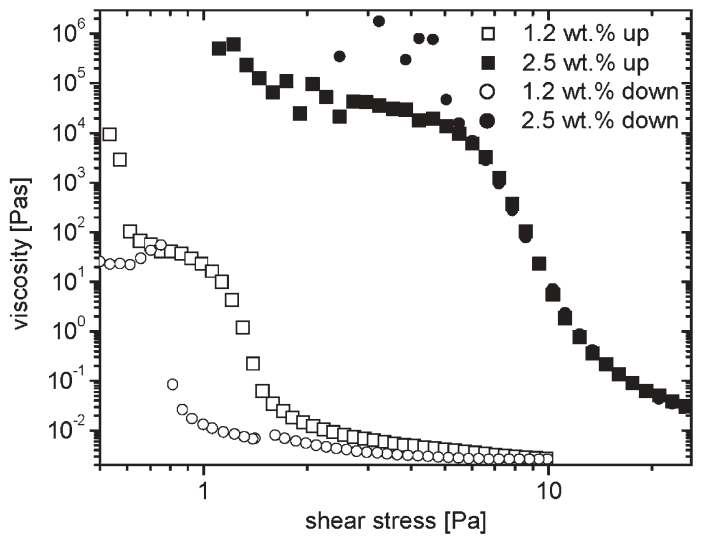

B

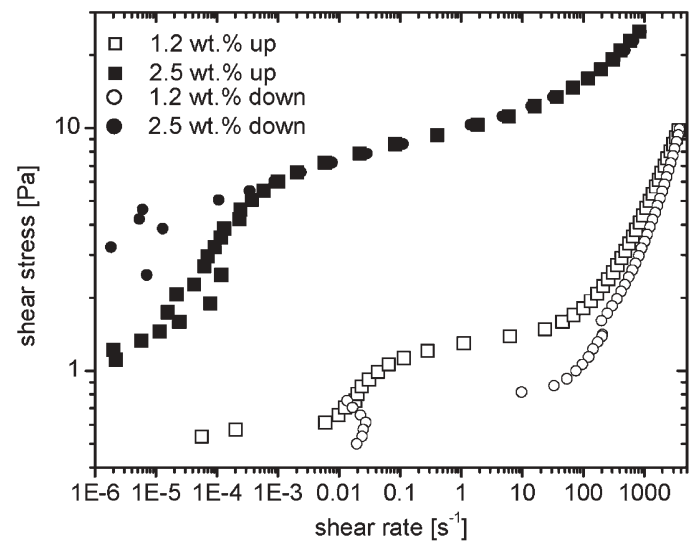

D

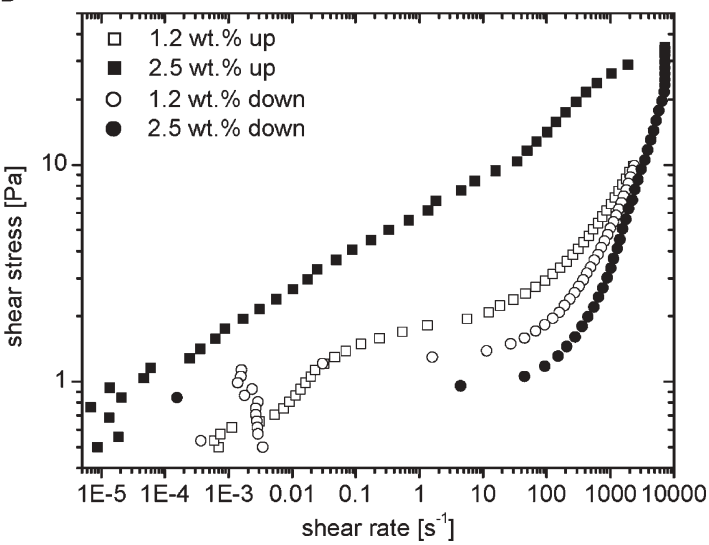

Fig. 13 Flow curves for boehmite at two different concentrations: (a) broken gel, shear stress-controlled shear rate; (b) broken gel, controlled shear stress-shear rate; (c) broken gel, viscosity-controlled shear stress; (d) recovered gel, controlled shear stress-shear rate.

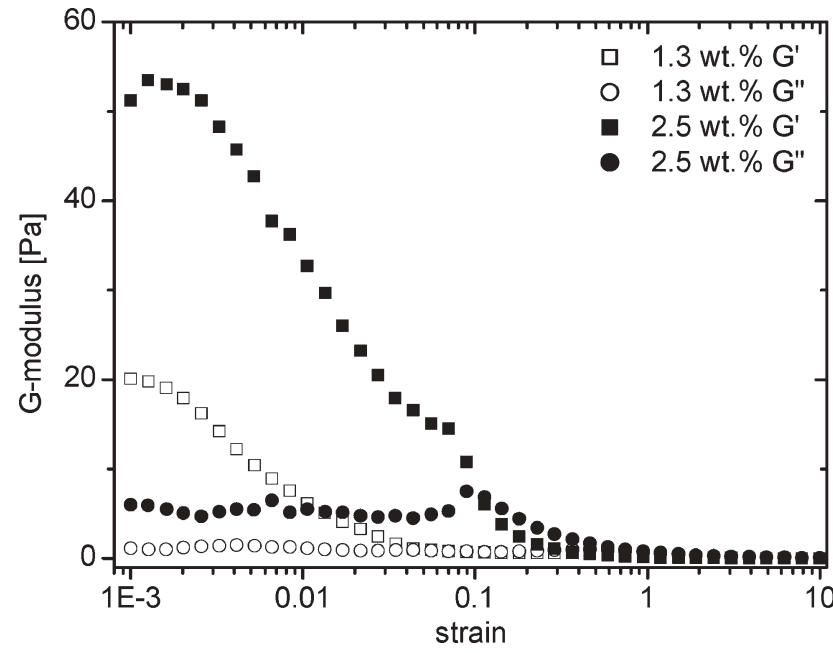

Fig. 14 Amplitude sweep for gibbsite broken gel at two different concentrations.

second key element is that we do not rely on a single technique, but apply a range of complementary rheological methods covering oscillatory, transient and steady shear measurements. These enable a wide range of deformational timescales and lengthscales to be explored, and a number of rheological parameters to be measured by different techniques. It has been

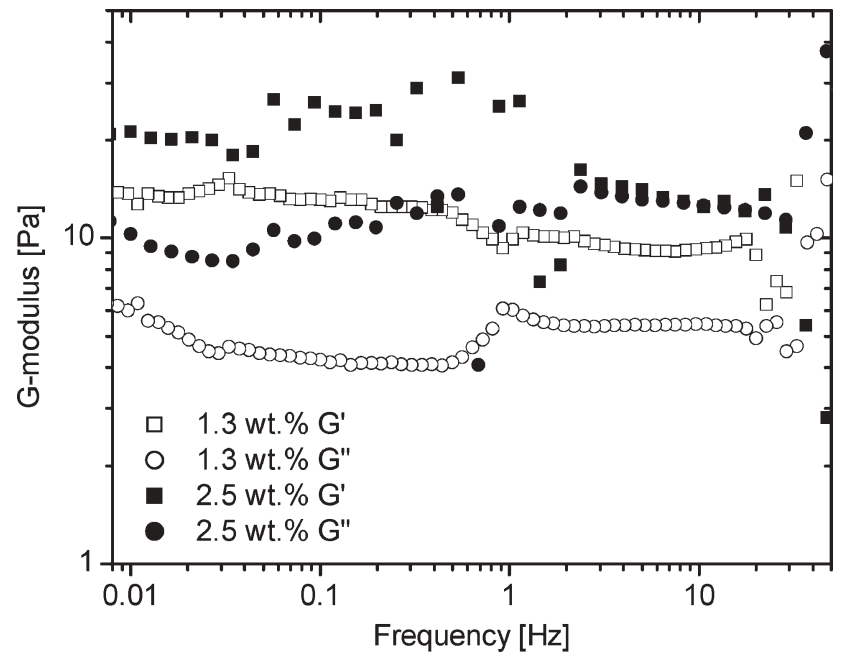

Fig. 15 Frequency sweep for gibbsite at two different concentrations for recovered gel.

demonstrated that even for these highly shear-sensitive materials, it is possible to obtain consistent values of parameters by alternative techniques, and to characterise their solid-liquid transition in a quantitative and reproducible manner. The pivotal role of creep measurements, which give low strain elastic and steady shear flow parameters in a single 

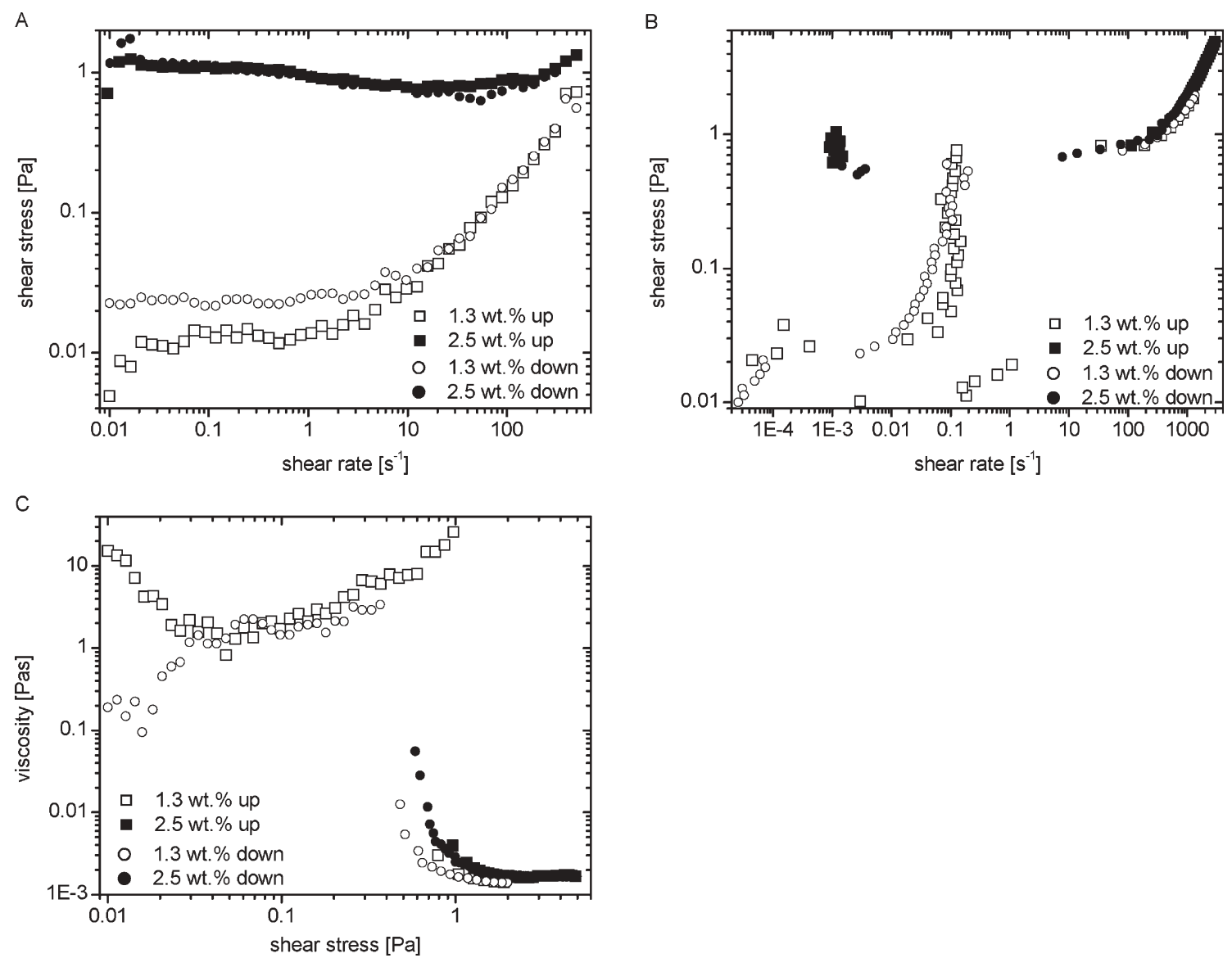

Fig. 16 Flow curves for gibbsite at two different concentrations: (a) broken gel shear stress-controlled shear rate; (b) broken gel controlled shear stress-shear rate; (c) recovered gel controlled shear stress-shear rate.

experiment, as the bridge between the linear and non-linear viscoelastic regimes and the link to other more restricted measurements, is clear. It is an essential tool for characterising soft solid materials.

Another advantage of this multi-technique approach is that the results from one method can be used to guide or validate complementary experiments. The strain amplitude sweep for oscillatory flow can define the limits of linear behaviour to ensure that storage-modulus measurements can be used to unambiguously monitor changes in gel characteristics rather than also contributing to those changes. It can also indicate the stress range and spacing that needs to be covered in creep measurements to traverse the whole range of behaviour from linear elasticity to fully developed flow. Clearly the methodology adopted here is a useful way to compare different rheological studies on a wide range of shear-sensitive materials.

We have compared the three different colloid dispersions at essentially the same mass concentrations in order to emphasise the effects of particle size and shape on the gelation properties. Industrial applications seek maximum exploitable effect (here the extent and rate of forming or destroying a gel) for minimum material use/cost. In the concentration range $1.2-2.5 \mathrm{wt} \%$, the hectorite and boehmite mineral colloids used both form quite strong gels, whereas gibbsite, largely as a result of its smaller maximum dimension and hence lower hydrodynamic volume (or higher $c^{*}$ ), forms relatively weak gels. However, we will see in part II that gelation effectiveness in mixed systems can be quite different from what is observed for pure component fluids.

Yet despite the wide range of gel intensity observed for these current fluids, a common picture of their rheology emerges which differs from the usual simple yield behaviour, widely published for many similar clay-based dispersions. ${ }^{33,34}$ Many studies report a monotonically increasing stress-strain rate flow curve, often appearing to extrapolate to a non-zero stress intercept at low strain rates. This is not observed for our systems until applied stresses of $1-10 \mathrm{~Pa}$ or strain rates above a few $s^{-1}$. It seems that the flow curves usually observed for montmorillonite, for instance, correspond to just the righthand (high stress and strain rate) branch of the present curves. Behaviour quite similar to that observed in this paper has previously been reported qualitatively by Burba and coworkers $^{40,41}$ and by Crabb et $a l^{42}$ for clay-mixed layer hydroxide systems.

At very low strains, all three systems studied here exhibit a frequency-independent plateau modulus which decreases slightly with increasing strain as the material both creeps and exhibits a very high low stress viscosity $\left(10^{4}-10^{6} \mathrm{~Pa} \mathrm{~s}\right)$, comparable with that of high molecular weight polymer melts. Unlike the latter, however, which typically maintain linear viscoelasticity until strains of 3-5 and high viscosities to shear 
rates of $1-10 \mathrm{~s}^{-1}$ or more, these colloidal soft solids become non-linear at strains $\gamma \ll 1$ and show dramatic shear thinning at shear rates $\ll 10^{-2} \mathrm{~s}^{-1}$. The stresses at which these sharp transitions in $G^{\prime}$ and $\eta$ occur range from $0.05-10 \mathrm{~Pa}$. However, the governing parameter appears to be shear strain; the critical strain seems reasonably invariant for each particle system, irrespective of concentration, varying from $0.1 \pm 0.05$ for gibbsite, through $0.3 \pm 0.05$ for hectorite to $0.45 \pm 0.05$ for boehmite. This suggests a structural model where once a critical local strain is exceeded, enabling local particle movement beyond some limit, the particle network rapidly fails cooperatively rather like the melting of a glass.

The colloidal particles used for this study have significant surface charges under the $\mathrm{pH}$ and low salinity conditions of the experiments (see Table 1). Hectorite is negatively charged, whereas both boehmite and gibbsite are positively charged. As pure components, they all form repulsive gels, whereby the overlapping electrical double layers of adjacent particles create repulsive forces between any given particle and its neighbours, causing a cooperative resistance to deformation and an elastic response to small applied stresses. (It is possible that the conditioning procedures used to prepare the hectorite suspensions could have released magnesium ions. ${ }^{43,44}$ Despite repeated washings, a small residual concentration of these divalent ions could have given rise to bridging attractive forces.) It was observed above that strain appears to be the controlling factor governing disruption of the gel, the critical strains being very small and in the order boehmite $(\sim 0.4)>$ hectorite $(\sim 0.3)>$ gibbsite $(\sim 0.1)$. This is the same order as the parameter $\alpha$, the ratio of the hydrodynamic volume of the particles to their real volume (see Table 1 ) $-\alpha$ increases from platelets to laths to rods - and this is also reflected in the order of $c^{*}$, the critical overlap concentration at which on average the particles start to overlap and the repulsive double layers have a major effect: boehmite $<$ hectorite $<$ gibbsite.

An additional set of parameters characterising the gels comes from the creep experiments in the form of the retardation times $\lambda_{1}$ and $\lambda_{2}$, which characterise the rate at which the systems creep following the application of a step stress. They are therefore a measure of the response times of the interacting particle network and can be looked on as experimental dynamic structural parameters. The times for the $2.5 \mathrm{wt} \%$ hectorite gel are displayed as a function of applied stress in Fig. 6. The timescales $(\sim 100 \mathrm{~s}$ and $\sim 10 \mathrm{~s})$ are typical of rotational relaxation times of laths/platelets of maximum dimension $\sim 300 \mathrm{~nm}$ in concentrated dispersions. It is interesting that the retardation times peak at $\tau \sim 2 \mathrm{~Pa}$; this is close to the stress where the creep viscosity also goes through a maximum. As the stress increases, the viscosity and shear modulus start to decrease gradually (see Fig. 7 for example) prior to the rapid critical decline at $\gamma_{\mathrm{y}}$ or $\tau_{\mathrm{y}}$. It can be seen that $\lambda_{1}$ and $\lambda_{2}$ also decrease as applied stress increases, more dramatically in fact than $\eta(t)$ or $G^{\prime}(\gamma)$; as the gel fails, one expects the motion of the colloidal particles to increase dramatically. Indeed, both $\lambda_{1}$ and $\lambda_{2}$ approach very low values as the observed yield stress for the hectorite gel $(8.5 \mathrm{~Pa})$ is approached, and this behaviour may in fact be used to estimate the critical breakdown stress. All this suggests that the creep retardation time spectrum can be used as a signature of the structural condition of the gel, and might in fact form a useful link between future microstructural models of colloidal gel rheology and experiment.

Once the critical failure strain is reached, the materials behave as viscoelastic liquids rather than elastoviscous gels, with a loss modulus at first comparable to and then dominating the storage modulus as strain increases, until the latter becomes negligible and viscous flow prevails. (We have not, however, investigated the presence of normal stresses in the steady shear experiments.) Although at first sight the materials seem to enter a post-yield, shear-thinning regime with viscosity decreasing in a power-law-like manner with increasing applied shear rate or stress, in fact on closer inspection they display very complex behaviour. For controlled shear rate, non-monotonic flow curves reminiscent of those observed for clay suspensions ${ }^{44}$ and viscoelastic wormlike micelle fluids ${ }^{37}$ are obtained, where the shear rate is a multi-valued function of stress and unstable flow regions with negative slope occur, within which shear-banding phenomena are expected ${ }^{45-48}$ and have been observed. ${ }^{38,49-54}$ This region is inaccessible when shear stress is the controlled variable; rapid jumps from the low shear-rate stable branch to the upper stable branch are expected as the applied stress reaches the extrema in the flow curves. Such extreme shear-thinning behaviour, exhibiting a power-law index of -1 , is indeed observed in these studies and the consistency between the flow paths observed in the two modes is compelling.

The fact that strain is the determining parameter for structural, and consequential rheological, changes for these soft materials is further demonstrated for the controlled strain rate, continuous shear experiments by plotting the measured stress versus accumulated strain. Fig. 17 is such a plot for the $2.5 \%$ hectorite broken gel at two values of the measurement time per point: $54 \mathrm{~s}$ and $200 \mathrm{~s}$. The measured stresses for the longer measurement time are higher, reflecting enhanced shear thickening for longer shearing times. However, the two curves have essentially the same shape and would roughly superimpose with a vertical shift factor of about 8 . For both curves the critical transitions occur at the same values of accumulated strain. The first maximum in stress occurs at a strain of 1-2,

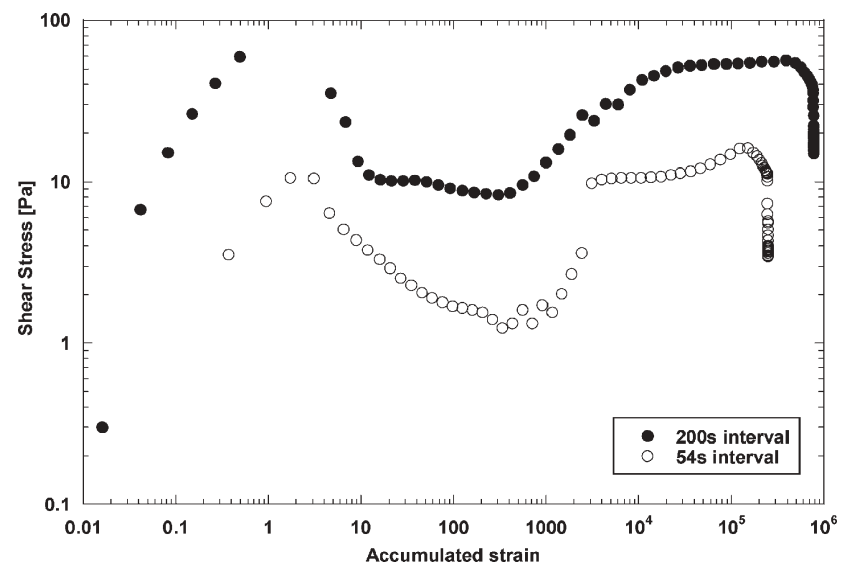

Fig. 17 Stress as a function of accumulated strain for two different measurement times per point in controlled shear-rate experiments on $2.5 \mathrm{wt} \%$ hectorite broken gel. 
similar if slightly higher to the values found in the earlier transient shear experiments. Similarly the subsequent stress minimum occurs at a strain of $\sim 400$ for both systems.

The controlled stress flow curves also exhibit two other features worthy of comment. At very low stresses and shear rates, a branch with negative slope is observed which exhibits a minimum in shear rate as stress increases before turning round to the more familiar increasing shear-rate behaviour at higher stresses. This corresponds to shear-thickening behaviour, likely due to shear-induced structural rearrangements reinforcing the interactions within the particle network prior to the disruptive effects of shear strain at higher stresses. Although it is possible that some of this effect is due to continued strengthening of the gel with time as it re-heals after the pre-shearing protocol, the short measurement times involved, the observation of this phenomenon for the $1000 \mathrm{~s}$ rested 'recovered gel' as well as for the less-healed 'broken gel', and the good reproducibility the curves, all suggest that we are observing significant shearthickening. Even if it were possible to carry out controlled shear-rate experiments at these very low flow rates (which cannot be performed in a controlled manner with this instrument), this unstable negatively sloping branch would not be observable.

The other unusual feature is usually associated with the controlled stress 'down' flow curve i.e. after the material has been subjected to the full range of stresses up to the maximum value for the 'up' stress ramp, the stress is then gradually decreased again with the same step sequence. The structurally disrupted fluid now re-heals as the stress decreases and superficially one might expect it to re-trace the 'up' curve. Initially this does indeed occur. However, the material is now rebuilding structure with a different stress-strain-time history than when it built structure at rest during the pre-shearing protocol and was subsequently modified during the 'up' stress ramp. So eventually it reaches a point where the balance between shear disruption and reinforcement of structure differs, and the 'down' flow curve deviates from the 'up' curve - the 'down' fluid is in fact a different structural material to its 'up' counterpart. For controlled shear rates, the flow curves deviate once the multi-modal shear-banding region (Cates, ${ }^{55}$ Cheng ${ }^{56}$ ) is reached. The 'down' curve tracks a different double-extrema path, with the extrema roughly mirroring the 'up' curve but displaced slightly and lower in amplitude. As shear rate is lowered, the gel strengthens and the rate of stress decrease becomes very low. With controlled shear stress, the behaviour is much as expected as the stress is lowered and the minimum in the controlled shear-rate curve is reached, causing a rapid jump to the stable lower branch. However, once this branch is reached, in many cases, particularly at higher concentrations, the shear-rate remains essentially constant as the stress continues to fall. Although the controlled shear-rate flow curve does not fall so rapidly with stress in this region, the controlled stress transition is noticeably sharper and suggests some abrupt re-structuring of the fluid in this region. This is unlikely to be due to wall slippage in the rheometer since the applied stress is decreasing and wall slip would decrease the platen rotation rate rather than maintain its value. This appears to be a structural relaxation phenomenon, with some annealing of the material as stress is reduced. Once the stress of the controlled rate-flow curve outer minimum is reached the shear rate rapidly decreases again, as the curve rejoins the flat outer branch and stresses are reached below which this restructured fluid hardly flows.

\section{A tentative physical model}

It is striking that the observed controlled stress flow curve, involving sharp transitions in shear rate at constant stress for both the increasing stress and decreasing stress branches, bears similarities to the inverted bifurcation phenomenon associated with the onset of convection in liquid mixtures. It has been shown (Lekkerkerker ${ }^{57}$ ) that this is caused by the competition between a stablising effect with a long relaxation time and a destabilising effect with a short relaxation time. In the present situation, we are observing the competing effects of rapid microstructural destabilisation by high shear-rate flows and slower stabilising orthokinetic and perikinetic restructuring processes.

Beyond this phenomenological analogy, this complex behaviour can be further rationalised in terms of a simple micro-structural model. Although somewhat speculative, we present this here as an attempt to give some insight into the underlying physics, which may be helpful in guiding further experiments and more quantitative models to probe and characterise this transitional region for colloidal gels. It is based on what we term 'flow-mediated building and disruption of structure.'

Fig. 18 shows a schematic representation of the typical shear stress-shear rate curves observed in this work. Let us consider what happens during a controlled shear rate continuous shear experiment, ramping up the shear rate from very low values. The initial gel, be it 'broken gel' or 'recovered gel', has been prepared by first shearing the system at a high shear rate for a significant time, to break down the colloidal gel network, its constituent weakly bound mesoscopic domains and hopefully also any more strongly aggregated particle tactoids. This hectorite suspension is then restored to rest and, as we have seen, gels quite quickly to give what we term a disordered gel $\left(\mathrm{G}_{\mathrm{I}}\right)$ - in its quiescent state the relative orientation of the lathlike particles is likely to be fairly random, reflecting much of

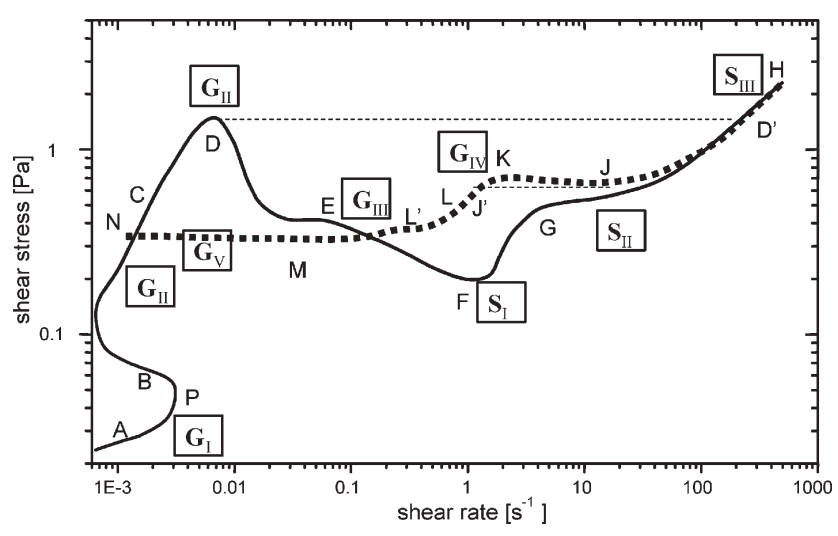

Fig. 18 Schematic diagram of controlled shear-rate-flow curves observed for hectorite, boehmite and gibbsite gels. Solid line: shear rate increasing from $10^{-3} \mathrm{~s}^{-1}$. Dashed line: shear rate decreasing from $500 \mathrm{~s}^{-1}$. 
the disorder of the highly-sheared liquid state from which it has been rapidly quenched, the only forces opposing the randomising Brownian motion being the weak electrostatic repulsions. As the fluid is sheared at low stresses-rates, initially the flow will have negligible effect on the material structure and the shear stress will increase with shear rate with a positive slope, reflecting the (high) viscosity of the disordered gel (region A Fig. 18). The stress-strain rate increases to a point where the shear can enhance the local alignment of the anisometric particles, so improving their packing and enhancing the strength of the gel and the measured stress. There is shear thickening of the material as it transforms to an ordered gel $\mathrm{G}_{\mathrm{II}}$ - regions $\mathrm{B}, \mathrm{C}$ (there may be some continuing gelation due to Brownian motion, but the similarity of the behaviour of the broken and recovered gels suggests that this effect is small compared to the shear-annealing effect in this region).

Eventually (region D) the strength of the shear energy becomes such that its main effect is to disrupt rather than create gel structure by moving portions of the particle network apart, in opposition to the structure inducing repulsive interparticle forces, causing the three-dimensional clay network to weaken and break down into discrete domains. Here we have a breaking gel $\mathrm{G}_{\mathrm{III}}$ (region $\mathrm{E}$ ), which with further disruption becomes a structured sol $\mathrm{S}_{\mathrm{I}}$ (region F). With increasing shear, the sol domains will be further broken down into the primary colloidal particles, although there will be some competition in this region with shear-induced (orthokinetic) restructuring. We therefore move into a breaking fluid $\left(\mathrm{S}_{\mathrm{II}}\right)$ regime reflected in the shear-thinning behaviour of region $\mathrm{G}$. In addition to disaggregation of the mesoscopic gelled domains and tactoids to dispersed hectorite platelets, at the highest shear conditions significant net alignment of the particles in the flow direction can also contribute to the shearthinning process (region $\mathrm{H}$ ). The limiting condition can be described as a dispersed, partially ordered sol, $\mathrm{S}_{\mathrm{III}}$.

Sol $\mathrm{S}_{\mathrm{III}}$ is the precursor state from which the initial broken gel' is quenched by immediately reducing the shear rate to zero. However, in measuring the flow curves, the final gel is now produced by a different route. The shear stress-strain-rate is reduced in stages and the fluid structure re-builds under a very different stress-strain-time locus than that in which it was broken down during the 'up' ramp. Initially in regions $\mathrm{H}$ and $\mathrm{G}$, the dispersed fluid restructures by closely re-tracing the conditions of the 'up' curve, with Brownian perikinetic restructuring proceeding as the orientating and destructuring power of the hydrodynamic forces reduces relative to thermally-induced collisions. However, eventually a condition is reached where the rate of shear-induced (orthokinetic) restructuring, due to hydrodynamic enhancement of the particle-collision frequency (which reduces linearly with $\dot{\gamma}$ ), exceeds that of shear degradation (which scales with the shear energy input $\sim \dot{\gamma}^{2}$ ) and for intermediate shear rates the restructuring rate is enhanced significantly above that due to Brownian effects alone. In region $\mathrm{J}$, there is a consequent upturn in the stress until, as both hydrodynamic effects on particle restructuring reduce in intensity, a maximum is reached at $\mathrm{K}$, followed by a pseudo-Newtonian region (region L, flow-curve slope $\sim 1$ ) in which the hydrodynamic and Brownian effects are essentially in balance. Hence the sol transforms to a gel over the region $\mathrm{J}-\mathrm{K}$ under conditions where there is still significant hydrodynamic alignment and ordering of the colloidal lath-like platelets and the gel in region $\mathrm{K}, \mathrm{G}_{\mathrm{IV}}$, can be termed a partially ordered gel. Beyond region L, where the 'down' curve crosses the 'up' curve, the hydrodynamic effects become increasingly weak and the stressstrain rate curve goes through a weak minimum, M, as the gelparticle network consolidates, through both continuing perikinetic Brownian structuring and the very low shear-induced annealing process identified earlier. The final ordered gel, $G_{V}$, becomes increasingly strong such that on the relatively short timescales of the continuous shear experiments (54 s per point), it displays an effective limiting yield stress, below which no flow can be observed. Longer measurement times or preferably creep experiments would be required to probe the deformational characteristics of this ordered gel, which is considerably stronger than the preconditioned 'broken' and 'recovered' gels whose properties and breakdown have been the main feature of this study.

Some comment is warranted as to how the observed flow curves fit into this mechanistic hypothesis and the general flow curve of Fig. 18. The controlled shear-stress experiments clearly display the negatively sloped region B (see Fig. 8b, 9b) and show that the extremum point $\mathrm{P}$ is $>\sim 10^{-3} \mathrm{~s}^{-1}$ (if region A exists, it occurs at significantly lower stresses than have been probed in these experiments). Region B will of course be unstable in controlled shear-rate experiments, the measured stress at shear rates corresponding to $\mathrm{P}$ and above jumping to the upper branch, region $\mathrm{C}$. The lowest shear rates plotted in Fig. 8a and 9a are the lowest values of $\dot{\gamma}$ for which stable stress measurements were obtained and all the curves show a steeply rising stress starting at shear rates just in excess of $10^{-3} \mathrm{~s}^{-1}$, in line with expectations (see especially the $2.5 \%$ recovered gel curve in Fig. 9a). These controlled shear-rate curves then display the maximum in stress, region $\mathrm{D}$, followed by all the other features of the 'up' curve in Fig. 18, regions E to H. Similarly, all the 'down' ramp curves in Fig. 8a and 9a display the features discussed in the mechanistic model, regions $J$ to $N$ and the various gel $\left(\mathrm{G}_{\mathrm{I}}-\mathrm{G}_{\mathrm{V}}\right)$ and sol $\left(\mathrm{S}_{\mathrm{I}}-\mathrm{S}_{\mathrm{III}}\right)$ states can be identified. Fig. 13a and 16a for boehmite and gibbsite, respectively, also show the same general characteristics.

Given this generic flow-curve behaviour, we can now give further interpretation to the controlled stress experiments. Taking the $1.5 \%$ hectorite recovered gel of Fig. $9 \mathrm{~b}$ as an example, because region $\mathrm{E}$ is unstable in controlled stress mode, the abrupt jump at $\tau \sim 1 \mathrm{~Pa}$ from an ordered gel $\mathrm{G}_{\mathrm{II}}$ at $\dot{\gamma}=5 \times 10^{-2}$ (D) to a disaggregating sol (breaking fluid) $\mathrm{S}_{\mathrm{II}}$ at $30 \mathrm{~s}^{-1}\left(\mathrm{D}^{\prime}\right)$ represents the effective yield stress of $\mathrm{G}_{\mathrm{II}}$. Regions $\mathrm{E}$ to $G$ are avoided and the shear rate jumps well into region $H$, whereafter this branch is traced out to the highest stresses measured. When the stress is reduced, branch $\mathrm{H}$ is essentially retraced until point $\mathbf{J}$ is reached $\left(\dot{\gamma} \sim 20 \mathrm{~s}^{-1}\right.$, see Fig. 9a). Since region $\mathrm{J}-\mathrm{K}$ is unstable in this mode, further stress reductions cause an abrupt reduction in shear rate to point $\mathbf{J}^{\prime}, \dot{\gamma} \sim 1 \mathrm{~s}^{-1}$. The stress at which this occurs, $\tau=1 \mathrm{~Pa}$, corresponds to the sol-gel transition stress, or effective yield stress, of the ordered gel $\mathrm{G}_{\mathrm{IV}}$. This gel has jumped into a region $\mathrm{L}$ where the hydrodynamic structuringordering of the fluid is reducing as stress falls, essentially linearly with $\tau$. So as the stress is reduced further below $1 \mathrm{~Pa}$, the gelnetwork ordering is reduced and this structural relaxation is accompanied by a corresponding linear reduction in viscosity. The 
corresponding shear rate therefore remains essentially constant. This process continues with further reductions in stress at constant shear rate, until the gel reaches the end of region $\mathrm{L}$ where hydrodynamic structuring effects are significant. At this point the gel structure and properties are determined only by the balance between hydrodynamic structure diasaggregation and Browniandriven network formation. The gel has essentially rejoined the curve $\left(\mathrm{L}^{\prime}\right.$ in Fig. 18) that it would have followed but for the intervention of hydrodynamic structuring in regions $\mathrm{J}-\mathrm{K}-\mathrm{L}$.

The flow-mediated building and disruption of fluid microstructures underlying this simple physical model can be incorporated into mathematical models of gel rheology, such as those introduced many years ago by Cheng and Evans, Moore and others, ${ }^{58,59}$ and more recently by Bonn et al. ${ }^{23}$ and by Cates et $a l^{45}$ The model of Bonn et al. ${ }^{23}$ has some of the features exhibited by the current systems, but only includes a flow-induced structure-disruption term. We speculate that the introduction of a competing flow-mediated structure-building term, operating on a different timescale, would give a model more in line with the behaviour of the hectorite and other gels described here.

This qualitative physical model is based on differences between the structuration and destructuration kinetics in different flow regimes characterised by homogeneous structural states. It could be envisaged that a non-homogeneous distribution of the particles across the measurement geometry could also be a contributory factor. However, in the absence of direct velocity-profile measurements, the fact that the results are essentially identical for $1^{\circ}, 2^{\circ}$ and $4^{\circ}$ cone angles for the cone-plate geometry, and are similar for a parallel plate geometry, suggest that the approach adopted here is valid. Nevertheless, the possibility of additional hydrodynamic instabilities cannot be ruled out, and studies of the evolution of both microstructure and micro-kinematics are essential aspects of furthering our understanding of the physical origins of these phenomena.

\section{Conclusions}

To summarise, these so-called viscoplastic materials display two distinct and relatively simple types of behaviour at the extremes of deformation. At extremely low strains, they behave as elastic(oviscous) solids with very high viscosity and at very large strains/strain-rates as shear-thinning low viscosity liquids. The transition region between these two conditions is extremely rich and complicated and cannot be described by a single parameter like a 'yield stress'. There is a 'yield space' which requires a range of parameters and timescales to describe it and which is a function of both the current structural state of the material and its stress-strain-time history. Yet with careful and complementary measurement procedures this yield space can be fully characterised.

The general characteristics of this 'yield space' have been quantified in terms of a range of parameters. The main parameters are low strain-low time-high frequency storage moduli $\left(G^{\prime}, G_{\mathrm{e}}\right)$, low stress viscosity $\eta(\tau \rightarrow 0)$, transition ('yield') stresses and strains $\left(\tau_{\mathrm{c}}, \tau_{\mathrm{y}}, \gamma_{\mathrm{c}}, \gamma_{\mathrm{y}}\right)$. It can be seen from Table 3 that the values obtained for the same sample by different techniques agree to within a factor of 2 , which is the order of reproducibility that we can expect for such fragile and shear-sensitive materials, and in line with the our experience of repeat experiments on both fresh and recycled samples. Strain appears to be the critical parameter for initial gel failure.

We have tried to capture the essential physics underlying this complex behaviour in a tentative qualitative model, based on flow-mediated building and disruption of structures. Two key issues now need to be addressed to build on this. On the one hand, combining rheological studies with structural probes, such as neutron and light scattering, is an essential part of improving our understanding of both the phenomenology and its underlying structural causes. On the other hand, the present qualitative ideas need to be translated into a quantitative model which can rationalise the observed diverse rheological behaviour.

Despite the same general behaviour of the three anisometric colloid types studied in this work, there are clear differences in the magnitudes of the stresses that they can sustain. These can be rationalised in terms of the differences in size and shape (aspect ratio) as they change from needle-like to lath-like and then plate-like. A subsequent paper will show even greater effects on gel characteristics and rheology when these colloids act synergistically in mixtures.

\section{Acknowledgements}

Annemieke J. T. ten Brinke was financially supported by the Nederlandse Organisatie voor Wetenschappelijk Onderzoek (NWO) and by Schlumberger Cambridge Research. The authors also acknowledge financial support from the SoftComp EU 6th Framework Network of Excellence, which enabled them to carry out exchange working visits as part of this research. They thank M. E. Cates, P. D. Olmsted and E. S. Boek for helpful discussions.

\section{References}

1 G. C. Maitland, "Rheology of montmorillonite gels", Proc. Xth International Congress of Rheology, International Congress of Rheology, Sydney, 1988, 140-142.

2 F. van der Kooij, H. N. W. Lekkerkerker and E. S. Boek, "Process Fluid", World Pat. WO 03/014252, 2003.

3 M. Moan, Physical Chemistry of Colloids and Interfaces in Oil Production, Institut Francais du Petrole, Reuil Malmaison, Paris, 1992, pp. 191-196.

4 H. A. Barnes, J. Non-Newtonian Fluid Mech., 1997, 70, 1-33.

5 A. M. Wierenga and A. P. Philipse, Colloids Surf., A, 1998, 137, 355-372; A. P. Philipse and A. M. Wierenga, Langmuir, 1998, 14, $49-54$.

6 B. C. Blakey and D. F. James, Colloids Surf., A, 2003, 231, 19-30.

7 J. C. Baird and J. Y. Walz, J. Colloid Interface Sci., 2006, 297, 161-169.

8 J. C. Baird and J. Y. Walz, J. Colloid Interface Sci., 2007, 306, 411-420.

9 M. Marquez, A. Robben, B. P. Grady and I. Robb, J. Colloid Interface Sci., 2006, 295, 374-387.

10 F. van der Kooij and H. N. W. Lekkerkerker, Langmuir, 2000, 16, 10144-10149.

11 S. M. Oversteegen, J. E. G. J. Wijnhoven, C. Vonk and H. N. W. Lekkerkerker, J. Phys. Chem. B, 2004, 108, 18158-18163.

12 S. M. Oversteegen, C. Vonk, J. E. G. J. Wijnhoven and H. N. W. Lekkerkerker, Phys. Rev. E: Stat., Nonlinear, Soft Matter Phys., 2005, 71, 041406.

13 S. K. Rhodes and J. A. Lewis, J. Am. Ceram. Soc., 2006, 89(6), $1840-1846$ 
14 A. A. Louis, E. Allahyarov, H. Wen and R. Roth, Phys. Rev. E: Stat., Nonlinear, Soft Matter Phys., 2002, 65(6), 061407.

15 J. L. Burba, W. E. Holman and C. R. Crabb, "Laboratory and Field Evaluation of Novel Inorganic Drilling Fluid Additive", Paper IADC/SPE 17198, IADC/SPE Drilling Conference, Society of Petroleum Engineers, Houston, Texas, 1988, pp. 179-186.

16 P. B. Laxton and J. C. Berg, J. Colloid Interface Sci., 2005, 285, $152-157$.

17 B. A. Firth and R. J. Hunter, J. Colloid Interface Sci., 1976, 57, 266-275.

18 D. D. Joye and G. W. Poehlein, Trans. Soc. Rheol., 1971, 15, $51-61$.

19 R. G. de Kretser, P. J. Scales and D. V. Boger, Colloids Surf., A, 1998, 137, 307-318

20 P. Coussot, Q. D. Nguyen, H. T. Huynh and D. Bonn, J. Rheol., 2002, 46, 573-589.

21 K. Bekkour, M. Leyana, A. Benchabaul and O. Scrivenor, J. Rheol., 2005, 49(6), 1329-1345.

22 P. B. Laxton and J. C. Berg, J. Colloid Interface Sci., 2006, 296, 749-755.

23 P. C. F. Møller, J. Mewis and D. Bonn, Soft Matter, 2006, 2, 274-283.

24 W. B. Russel and P. R. Sperry, Prog. Org. Coat., 1994, 23, 305-324.

25 I. M. Krieger and T. J. Dougherty, Trans. Soc. Rheol., 1959, 3, $137-152$.

26 C. G. de Kruif, E. M. F. van Iersel, A. Vrij and W. B. Russel, J. Chem. Phys., 1985, 83, 4717-4725.

27 V. Trappe and P. Sandbuler, Curr. Opin. Colloid Interface Sci., 2004, 8, 494-500.

28 G. Petekidis, D. Vlassopoulos and P. N. Pusey, J. Phys.: Condens. Matter, 2004, 16, S3955-S3963.

29 G. R. Gray and H. C. H. Darley, Composition and Properties of Oil Well Drilling Fluids, Gulf, Houston, 5th edn, 1988.

30 I. C. Callaghan and R. H. Ottewill, Faraday Discuss. Chem. Soc., 1974, 57, 110-118.

31 L. M. Barclay and R. H. Ottewill, Spec. Discuss. Faraday Soc., 1970, 1, 138-147.

32 S. D. Lubetkin, S. R. Middleton and R. H. Ottewill, Philos. Trans. R. Soc London, Ser. A, 1984, A311, 353-368.

33 S. Abend and G. Lagaly, Appl. Clay Sci., 2000, 16, 201-227.

34 L. J. Michot, I. Bihannic, K. Porsch, S. Maddi, C. Baravian, J. Mongel and P. Levitz, Langmuir, 2004, 20, 10829-10837.

35 M. P. B. van Bruggen, Langmuir, 1998, 14, 2245-2255.

36 A. M. Wierenga, T. A. J. Lenstra and A. P. Philipse, Colloids Surf., A, 1998, 134, 359-371.

37 J.-F. Berret, Langmuir, 1997, 13, 2227-2234.

38 Y. T. Hu, P. Boltenhagen and D. J. Pine, J. Rheol., 1998, 42, $1185-1208$.
39 F. Da Cruz, F. Chevoir, D. Bonn and P. Coussot, Phys. Rev. E: Stat., Nonlinear, Soft Matter Phys., 2002, 66, 051305.

40 J. L. Burba and A. L. Barnes, "Mixed metal layered hydroxide-clay adducts as thickeners for water and other hydrophilic fluids.", US Pat. 4664843, 1987.

41 J. L. Burba, W. F. Tehan, F. D. Hamilton, W. E. Holman, C. Porzucek and C. P. Christenson, "Field evaluations confirm superior benefits of $\mathrm{MMH}$ field system on hole cleaning, borehole stability and rate of penetration", Paper IADC/SPE 19956, IADC/ SPE Drilling Conference, Society of Petroleum Engineers, Houston, Texas, 1990, pp. 371-380.

42 C. R. Crabb, J. L. Burba and W. E. Holman, "New inorganic attapulgite extender provides stable rheology and supports heavy cuttings under severe conditions", Paper SPE 18482, SPE International Symposium on Oilfield Chemistry, Society of Petroleum Engineers, Houston, TX, 1989, pp. 219-225.

43 A. Mourchid and P. Levitz, Phys. Rev. E: Stat. Phys., Plasmas, Fluids, Relat. Interdiscip. Top., 1998, 57, R4887-4890.

44 F. Pignon, A. Magnin and J. M. Pau, J. Rheol., 1996, 40, 573-587.

45 N. Spenley, M. E. Cates and T. C. B. McLeish, Phys. Rev. Lett., 1993, 71, 939-942.

46 P. D. Olmsted, Europhys. Lett., 1999, 48, 339-345.

47 J. K. G. Dhont, Phys. Rev. E: Stat. Phys., Plasmas, Fluids, Relat. Interdiscip. Top., 1999, 60, 4534.

48 C.-Y. David Lu, P. D. Olmsted and R. C. Ball, Phys. Rev. Lett., 2000, 84, 642-645.

49 M. E. Britton and P. T. Callaghan, Phys. Rev. Lett., 1997, 78, 4930-4933.

50 J. P. Decruppe, S. Lerouge and J. F. Berret, Phys. Rev. E: Stat., Nonlinear, Soft Matter Phys., 2001, 63, 022501.

51 P. Coussot, J. S. Raynaud, F. Bertrand, P. Moucheront, J. P. Guilbaud, H. T. Huynts, S. Jarny and D. Leseur, Phys. Rev. Lett., 2002, 88, 218301.

52 J. B. Salmon, A. Colin and S. Manneville, Phys. Rev. Lett., 2003, 90, 228303

53 A. Ragouilliaux, B. Herzhaft, F. Bertrand and P. Coussot, Rheol. Acta, 2006, 46, 261-271.

54 K. Kang, M. P. Lettinga, Z. Dogic and J. K. G. Dhont, Phys. Rev. E: Stat., Nonlinear, Soft Matter Phys., 2006, 74, 026307.

55 M. E. Cates, NATO ASI Ser., Ser. E, 1997, 272-276.

56 D. C.-H. Cheng, Rheol. Acta, 2002, 42, 372-382.

57 H. N. W. Lekkerkerker, in Light Scattering in Liquids and Macromolecular Solutions, ed. V. Degiorgio, M. Corti and M. Giglio, Plenum Press, New York, 1980, 231-241.

58 D. C.-H. Cheng and F. Evans, Br. J. Appl. Phys., 1965, 16, $1599-1617$.

59 F. Moore, Br. Ceram. Soc. Trans., 1959, 58, 470-484; M. M. Cross, J. Colloid Sci., 1965, 20, 417-437. 